\title{
La desigualdad en Centroamérica durante el decenio de 1990
}

\author{
Juan Diego Trejos y Thomas H. Gindling.
}

Juan Diego Trejos,

Profesor de la Universidad de Costa Rica

e investigador del Instituto de

Investigaciones en Ciencias Económicas

de la Universidad de Costa Rica

$\bullet$ jdtrejos@cariari.ucr.ac.cr

T. H. Gindling

Profesor, Universidad de Maryland en

Baltimore County

e investigador visitante del Instituto de Investigaciones

en Ciencias Económicas de la

Universidad de Costa Rica

$\infty$ tgindlin@umbc.edu

Este trabajo busca respuesta a dos preguntas: ¿cómo y por qué cambió la distribución de los ingresos laborales en Centroamérica? y ¿por qué Costa Rica presenta una mayor equidad? Para responderlas se usa una técnica basada en la estimación de ecuaciones de remuneración. La dirección de los cambios en la desigualdad no es uniforme y depende del indicador utilizado. Aunque sólo Costa Rica y Guatemala muestran un claro deterioro, se encuentran fenómenos comunes en todos los mercados de trabajo, donde el aumento en la dispersión de las jornadas causa el mayor impacto negativo en la distribución. Dos son las causas principales de la menor desigualdad relativa en Costa Rica: la educación se distribuye de manera más igualitaria y las diferencias salariales entre las zonas son más bajas. Esto significa que la universalización de la educación primaria y la provisión de infraestructura económica y social a las comunidades rurales permiten reducir la desigualdad. 


\section{I}

\section{Introducción}

Luego del convulso decenio de 1980, caracterizado por la crisis de la deuda, la inestabilidad política y los conflictos armados, los países de Centroamérica iniciaron un conjunto de reformas económicas que acompañaron y complementaron los procesos de pacificación y de restauración de la democracia en varias de sus economías. Estas reformas fueron apoyadas por los organismos financieros internacionales y por las agencias pertinentes de los países donantes, particularmente la de los Estados Unidos, y se enmarcaron, con diverso énfasis e intensidad, dentro del llamado consenso de Washington.

Las reformas implicaron abandonar el estilo de desarrollo que había predominado durante los tres decenios anteriores y que se caracterizó por una industrialización sustitutiva de las importaciones en el marco de un mercado común regional, aunque con amplias diferencias entre países en cuanto a políticas sociales y distributivas y a la situación inicial. Este estilo de desarrollo fue muy exitoso en términos de crecimiento en la década de 1960, pero empezó a mostrarse inviable durante el decenio de 1970 y colapsó con la crisis de la deuda a comienzos de 1980. Costa Rica fue el país que inició más tempranamente las reformas económicas, a mediados del decenio de 1980, seguido de Guatemala. Los demás países centroamericanos sólo avanzaron en tales esfuerzos a partir del decenio de 1990.

La década de 1990 mostró el reinicio del crecimiento económico en la región y la consolidación, lenta e incompleta, de un nuevo estilo de desarrollo hacia afuera, cuyo motor principal eran las exportaciones extrarregionales. El objetivo de este trabajo es el de analizar los cambios distributivos que tuvieron lugar en ese decenio de reformas económicas y pacificación y buscar las causas que explican las diferencias entre países y su evolución. Para cumplirlo, se hace hincapié en los ingresos del trabajo, principal componente del ingreso de las familias, y se estudian las causas de su distribución y de su evolución. Se concluye recapitulando los principales hallazgos e identificando sus implicaciones de política.

\section{II}

\section{El contexto: una región pequeña pero heterogénea}

Centroamérica es una región pequeña en tamaño, población y capacidad económica. Como indica el cuadro 1 , su territorio alcanza a los $432.000 \mathrm{~km}^{2}$, lo que equivale a cerca de una cuarta parte del territorio mexicano y a apenas la décima parte del estadounidense. En el año 2000 su población global se aproximaba a los 33 millones de habitantes, cerca del $6 \%$ de la población latinoamericana. Su producción total ese año llegó a los 50.000 millones de dólares de 1995 , lo que representa el 3\% de la producción latinoamericana, equivale al $11 \%$ de la producción de México y a menos de $1 \%$ de la estadounidense. Al interior de la región, Nicaragua es el país más grande, en tanto que Guatemala concentra la mayor población y genera la producción total más alta. El Salvador es el más pequeño, pero el más densamente poblado, mientras que Costa Rica muestra el menor tamaño poblacional. La mitad de la población centroamericana continúa residiendo en las zonas rurales, pese a que en El Salvador y Nicaragua empieza a predominar levemente la población urbana.

La subregión centroamericana también es muy heterogénea. Utilizando el indicador más tradicional de desarrollo económico relativo - el producto interno bruto (РІB) per cápita en dólares de 1995-, en el año 2000 Costa Rica duplicó el promedio regional y estuvo por encima de los 3.600 dólares. El Salvador y Guatemala se ubicaron en torno al promedio regional, con un PIB per cápita cercano a los 1.600 dólares anuales, mientras que Honduras y Nicaragua 
CUADRO 1

Centroamérica: Indicadores sobre su situación socieconómica y evolución reciente, por país

\begin{tabular}{|c|c|c|c|c|c|c|}
\hline Indicador & Costa Rica & El Salvador & Guatemala & Honduras & Nicaragua & Región \\
\hline Población total año 2000 (millones) & 4,0 & 6,3 & 11,4 & 6,5 & 5,1 & 33,2 \\
\hline Distribución subregional (\%) & 12 & 19 & 34 & 20 & 15 & 100 \\
\hline Crecimiento anual, 2000/1990 & 2,8 & 2,1 & 2,7 & 2,9 & 2,9 & 2,6 \\
\hline Porcentaje que reside en zonas urbanas & 50 & 60 & 40 & 53 & 56 & 51 \\
\hline Territorio total (miles de $\mathrm{km}^{2}$ ) & 50,9 & 20,9 & 108,9 & 112,1 & 139,0 & 431,8 \\
\hline Densidad (personas por $\mathrm{km}^{2}$ ) & 79 & 300 & 105 & 58 & 37 & 77 \\
\hline Producción año 2000 (millones de dólares de 1995) & 14774 & 10978 & 17695 & 4595 & 2446 & 50488 \\
\hline Distribución subregional (\%) & 29 & 22 & 35 & 9 & 5 & 100 \\
\hline Crecimiento anual 2000/1990 & 4,7 & 4,3 & 4,1 & 3,1 & 3,5 & 4,3 \\
\hline Producción per cápita año 2000 (dólares de 1995) & 3672 & 1749 & 1554 & 709 & 482 & 1519 \\
\hline Índice promedio subregional $=100$ & 242 & 115 & 102 & 47 & 32 & 100 \\
\hline Desarrollo social en $2000^{a}$ & 0,820 & 0,706 & 0,631 & 0,638 & 0,635 & 0,670 \\
\hline Posición entre 173 países & 43 & 104 & 120 & 116 & 118 & 112 \\
\hline Tendencia 1990 a 2000 & Mejoró & Mejoró & Mejoró & Mejoró & Mejoró & Mejoró \\
\hline Gasto social per cápita 1999 (dólares de 1997) & 622 & 82 & 107 & 57 & 57 & 147 \\
\hline Prioridad fiscal (\% del gasto público) & 43 & 27 & 46 & 34 & 37 & 38 \\
\hline Prioridad macroeconómica (\% del PIB) & 17 & 4 & 6 & 7 & 13 & 8 \\
\hline Tendencia 1990 a 1999 & Aumentó & Aumentó & Aumentó & Disminuyó & Aumentó & Aumentó \\
\hline \multicolumn{7}{|l|}{ Pobreza hacia 1999} \\
\hline Personas pobres $(\%)$ & 20 & 50 & 61 & 80 & 70 & 59 \\
\hline Tendencia 1990 a 1999 & Disminuyó & Disminuyó & Disminuyó & Sin cambio & Disminuyó & Disminuyó \\
\hline \multicolumn{7}{|l|}{ Desigualdad ingreso total hacia 1999} \\
\hline Tendencia 1990 a 1999 & Aumentó & Aumentó & Sin cambio & Disminuyó & Sin cambio & $\ldots$ \\
\hline \multicolumn{7}{|l|}{ Mercado de trabajo hacia 1999} \\
\hline Tasa neta de participación, hombres & 75 & 72 & 81 & 80 & 79 & 78 \\
\hline Tendencia 1990 a 1999 & Disminuyó & Disminuyó & Disminuyó & Aumentó & Aumentó & $\ldots$ \\
\hline Tasa neta de participación, mujeres & 36 & 42 & 42 & 41 & 39 & 40 \\
\hline
\end{tabular}

Fuente: Elaboración propia con base en datos de la CEPAL (2001, 2002 y 2003), PNUD (2002) y Trejos (2002).

a Índice de desarrollo humano (IDH) del PNUD.

se ubicaron debajo de la mitad del promedio zonal, con PIB per cápita inferiores a 700 dólares. Estas diferencias se han mantenido, aunque se aminoran cuando se expresan en paridad de poder de compra (PNUD, 2002) y, como se observa en el gráfico 1, no se han experimentado modificaciones sustantivas en los últimos veinte años.

La heterogeneidad también se expresa en el desarrollo social y ello es fruto de una inversión social que también muestra amplias disparidades y que refleja y refuerza el nivel del ingreso nacional y las disímiles políticas distributivas. Costa Rica ostenta el
PIB per cápita más alto, el mayor desarrollo social, ${ }^{1}$ la menor desigualdad y la incidencia de la pobreza más baja, todo lo cual se sustenta en una inversión social de larga data y una alta prioridad macroeconómica y fiscal; como se verá posteriormente, este país muestra las sinergias que se obtienen al invertir en capital humano: mayor productividad, menor desigualdad y menor pobreza. El Salvador presenta una situación

\footnotetext{
${ }^{1}$ Medido por el Índice de Desarrollo Humano (IDH) del Programa de las Naciones Unidas para el Desarrollo (PNUD).
} 
GRAFICO 1

Centroamérica: Evolución de la producción real por habitante (PIB per cápita en dólares de 1995)

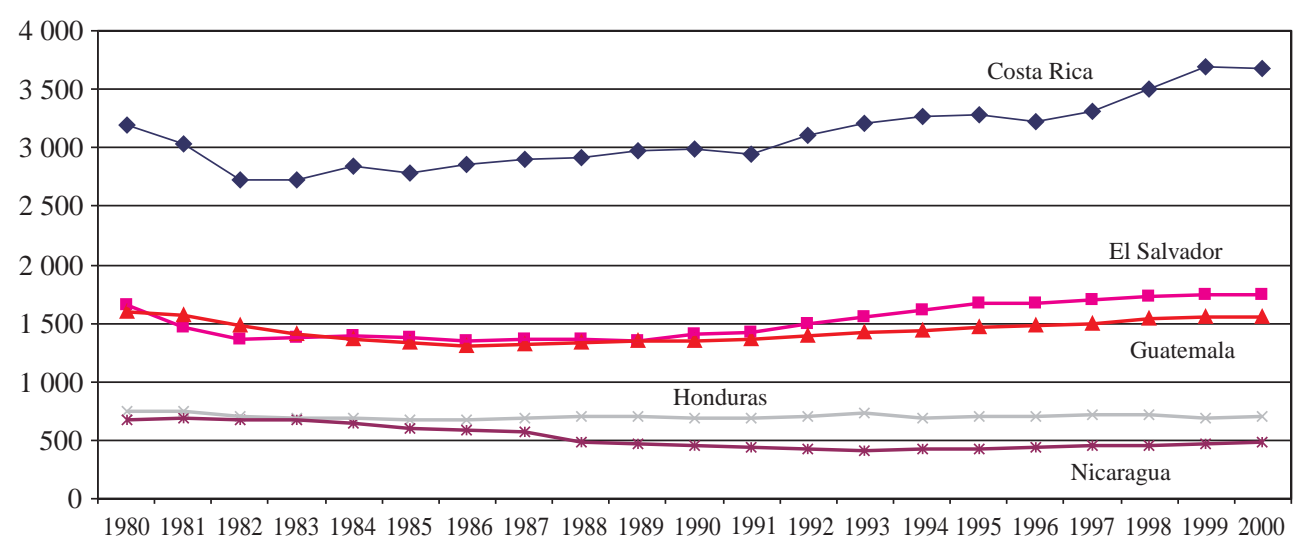

Fuente: CEPAL (2003).

intermedia en cuanto a pobreza, desigualdad y desarrollo social, en tanto que los tres países restantes se hallan entre los países latinoamericanos que ostentan los mayores niveles de pobreza y de desigualdad (CEPAL, 2002). ${ }^{2}$

La heterogeneidad de la subregión se observa también en el mercado de trabajo. La participación laboral es mayor en los países más pobres y ha aumentado en todos ellos gracias a una creciente incorporación de las mujeres. Aun así, y excepto en El Salvador, la participación femenina continúa siendo la mitad de la masculina. La incidencia del desempleo abierto va desde un $2 \%$ para Guatemala hasta un $12 \%$ para Nicaragua. Sólo Costa Rica muestra un leve aumento del desempleo, manteniéndose éste sin cambios en el resto de los países, salvo en Nicaragua, donde presenta una reducción a casi la mitad de la alta tasa mostrada a inicios de la década. Esta diversidad también se observa en la composición y característica del empleo generado. Costa Rica, y en menor medida El Salvador, disfrutan de un mercado de trabajo más formalizado, con un menor peso de las actividades agrícolas y de baja productividad (agricultura tradicional y sector informal), una mayor presencia de relaciones salariales y una fuerza de trabajo un tanto más calificada. Sin

\footnotetext{
${ }^{2}$ La subregión es muy vulnerable a desastres naturales. Estos fenómenos han afectado en mayor medida a los países más pobres, como lo hizo el conocido huracán Micth de 1998. También varios países centroamericanos sufrieron graves conflictos armados, apoyados por las fuertes desigualdades existentes, que ampliaron las brechas entre ellos.
}

embargo, si se mira la subregión en su conjunto, un tercio del empleo sigue vinculado a las actividades agrícolas, la mitad de los ocupados se inserta en actividades de baja productividad, un $42 \%$ desarrolla actividades por cuenta propia o como familiar no remunerado y la mitad de los trabajadores no ha completado los primeros seis años de educación primaria.

Luego de una contracción generalizada de la producción por habitante en el decenio de 1980, la subregión creció en la década de 1990 en forma bastante sostenida, a un ritmo medio anual de 4,2\% (y de 1,6\% per cápita), con aumentos en la inversión social real y mejora en los indicadores de desarrollo social (cuadro 1 y gráfico 1). Pese a ello, Guatemala, Honduras y Nicaragua no han logrado recuperar el nivel de producción alcanzado 20 años atrás; los países que menos mejoraron fueron los que estaban en peor situación al inicio de la década de 1990, indicando así una ampliación de las brechas o heterogeneidades internas.

Pese a que todos los países de la subregión han emprendido reformas económicas, ha habido diferencias en el momento de aplicarlas, en sus características y en sus resultados más generales. Costa Rica fue el país que comenzó más tempranamente las reformas económicas (1985/1986), seguido de Guatemala (1986/ 1987), Honduras (1990), Nicaragua (1993) y El Salvador (1993/1994). Todos iniciaron procesos de apertura comercial, liberalización financiera y reformas fiscales. Salvo Costa Rica, todos avanzaron en un programa importante de privatización, mientras ninguno efectuó reformas significativas en la legislación 


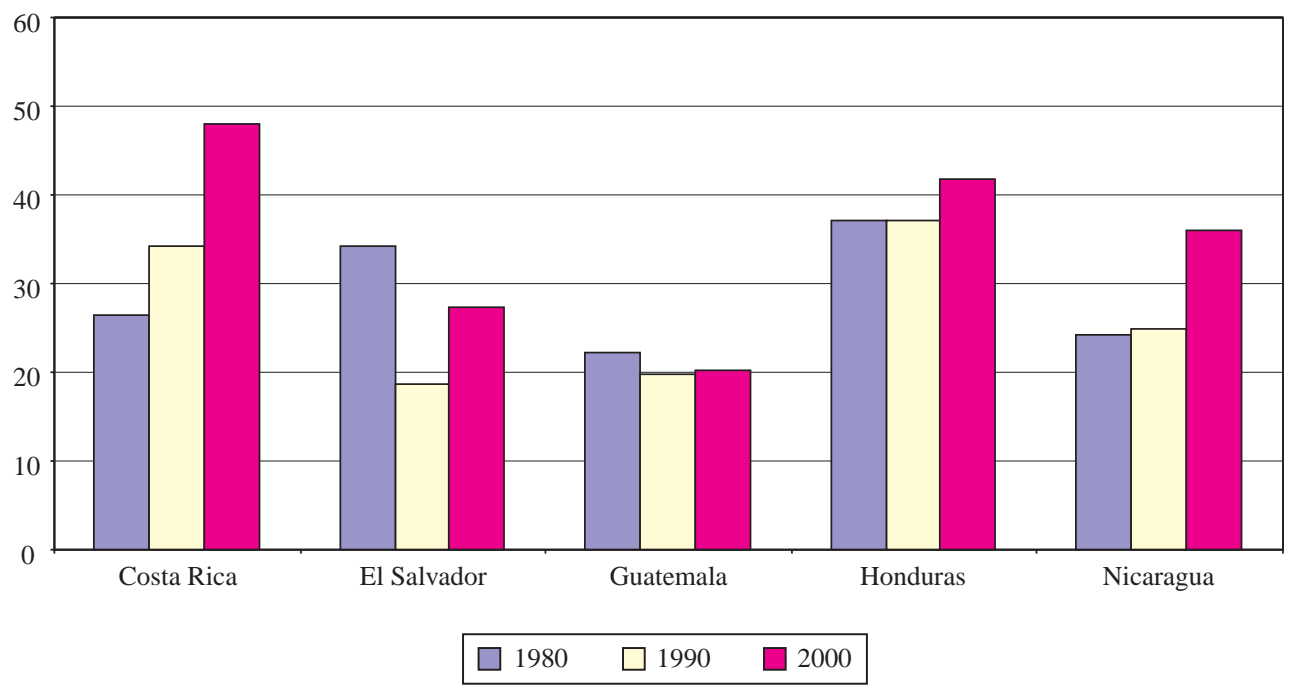

Fuente: CEPAL (2003).

laboral. ${ }^{3}$ El objetivo de las reformas aplicadas fue reorientar la producción desde el mercado interno o regional hacia el resto del mundo. El gráfico 2 muestra que en la década de 1980 las exportaciones, como porcentaje del PIB, sólo aumentaron considerablemen- te en Costa Rica, primer país de la subregión en iniciar las reformas. En la década de 1990 la importancia de las exportaciones en los otros países de la región también empezó a aumentar, especialmente en El Salvador y Nicaragua, vinculada con la maquila.

\section{III}

\section{Las fuentes de la desigualdad en los ingresos laborales}

Para estudiar las causas de las diferencias en materia de desigualdad entre países y en el tiempo se analiza la distribución de los ingresos laborales. ${ }^{4}$ Esto se hace

\footnotetext{
${ }^{3}$ En cuanto a medidas de la magnitud de las reformas en América Latina, véase Lora (2001) y Morley, Machado y Pettinato (1999).

${ }^{4}$ Para tener la visión más completa posible de la evolución distributiva en el decenio de 1990, hemos considerado las estimaciones disponibles más próximas al inicio y fin del decenio para cada país. Las encuestas que utilizamos fueron: para Costa Rica, las Encuestas de Propósitos Múltiples de 1990 y 1999; para El Salvador, las Encuestas de Propósitos Múltiples de 1995 y 1999; para Guatemala, la Encuesta Sociodemográfica de 1989 y la Encuesta de Ingresos y Gastos de 1998; para Honduras, las Encuestas Permanentes de Hogares de Propósitos Múltiples de 1990 y 1999; y para Nicaragua, las Encuestas Nacionales de Hogares sobre Me-
}

por tres razones. Primero, mientras las encuestas utilizadas miden los ingresos familiares totales con diferencias de cobertura en los rubros medidos en cada país y año, los datos sobre ingresos laborales se miden de manera más homogénea en cada país y año y son de mejor calidad.

Segundo, en general los ingresos del trabajo aportan una proporción muy alta de los ingresos totales y de la desigualdad (CEPAL, 2002). Por ejemplo, Székely

dición de Niveles de Vida de 1993 y 1998. Las encuestas se levantan en los meses de julio (Costa Rica), septiembre (Honduras), febrero a junio (Nicaragua 1993), abril a septiembre (Nicaragua 1998), y durante todo el año (El Salvador y Guatemala). 
Centroamérica: Indicadores de la desigualdad de los ingresos laborales, por país, a fines del decenio de 1990

(Para ocupados de 15 o más años con ingreso y horas trabajadas conocidas)

\begin{tabular}{|c|c|c|c|c|c|}
\hline $\begin{array}{l}\text { Medida de desigualdad y grupo } \\
\text { de ocupados }\end{array}$ & $\begin{array}{l}\text { Costa Rica } \\
1999\end{array}$ & $\begin{array}{l}\text { El Salvador } \\
1999\end{array}$ & $\begin{array}{l}\text { Guatemala } \\
1998\end{array}$ & $\begin{array}{l}\text { Honduras } \\
1999\end{array}$ & $\begin{array}{l}\text { Nicaragua } \\
1998\end{array}$ \\
\hline \multicolumn{6}{|l|}{ Todos los trabajadores } \\
\hline Coeficiente de Gini & 0,436 & 0,469 & 0,577 & 0,543 & 0,562 \\
\hline Índice de Theil & 0,347 & 0,412 & 0,701 & 0,583 & 0,705 \\
\hline Varianza del logaritmo del ingreso & 0,775 & 0,779 & 1,436 & 1,203 & 1,039 \\
\hline \multicolumn{6}{|l|}{ Trabajadores asalariados } \\
\hline Coeficiente de Gini & 0,406 & 0,433 & 0,502 & 0,465 & 0,494 \\
\hline Índice de Theil & 0,306 & 0,338 & 0,502 & 0,427 & 0,528 \\
\hline Varianza del logaritmo del ingreso & 0,620 & 0,644 & 0,964 & 0,774 & 0,669 \\
\hline \multicolumn{6}{|l|}{ Asalariados, excepto serv. doméstico } \\
\hline Coeficiente de Gini & 0,388 & 0,419 & 0,499 & 0,450 & 0,486 \\
\hline Indice de Theil & 0,282 & 0,318 & 0,499 & 0,403 & 0,506 \\
\hline Varianza del logaritmo del ingreso & 0,529 & 0,599 & 0,927 & 0,705 & 0,643 \\
\hline \multicolumn{6}{|l|}{ Trabajadores de la zona urbana } \\
\hline Coeficiente de Gini & 0,435 & 0,461 & 0,560 & 0,495 & 0,546 \\
\hline Índice de Theil & 0,337 & 0,402 & 0,630 & 0,484 & 0,647 \\
\hline Varianza del logaritmo del ingreso & 0,774 & 0,777 & 1,308 & 0,926 & 0,949 \\
\hline
\end{tabular}

Fuente: Cálculos de los autores con base en las encuestas de hogares de los países y años respectivos.

y Hilgert (1999a) calculan que los ingresos laborales son responsables en una alta proporción de la desigualdad del ingreso total: del 83\% en Costa Rica (1997), del $76 \%$ en El Salvador (1995) y del $73 \%$ en Guatemala (1998). La desigualdad asociada con los ingresos laborales también puede explicar una alta proporción de los cambios en la desigualdad del ingreso total. Según Székely y Hilgert (1999b), los cambios asociados con el ingreso laboral pueden explicar el 100\% o más del cambio en la desigualdad del ingreso total en Costa Rica (1989-1997), en El Salvador (19951998) y en Honduras (1989-1998).

Finalmente, la teoría económica, y en particular el avance de las técnicas econométricas, permiten analizar mejor las causas de los cambios en la distribución de los ingresos laborales. Con el fin de examinar la desigualdad de tales ingresos se puede hacer referencia a las teorías de la economía del trabajo (por ejemplo, la teoría del capital humano) y las técnicas econométricas (por ejemplo, ecuaciones de remuneración).

\section{La desigualdad de los ingresos laborales a fi- nes del decenio de 1990}

Para analizar la desigualdad de los ingresos laborales mensuales se uniforma la población de los países, con- siderando sólo a los ocupados de 15 años o más que informaron de ingresos y horas trabajadas. Esto implica excluir a los menores de 15 años en todos los países, y a los trabajadores familiares no remunerados, que son importantes en Guatemala, Honduras, Nicaragua y, en menor medida, en El Salvador. También se excluyen los trabajadores que no reportaron ingresos. Lo último es importante en Costa Rica, especialmente en el caso de los trabajadores independientes, que estarían subrepresentados en este análisis. ${ }^{5}$

El cuadro 2 resume tres indicadores de desigualdad relativa para los ocupados de 15 años o más y con ingresos conocidos, así como para subgrupos de ellos. El coeficiente de Gini es más sensible a los cambios en la parte media de la distribución, el índice de Theil aporta información sobre cambios en la parte alta de la distribución, en tanto que la varianza del logaritmo del ingreso es más sensible a lo que sucede en la parte baja de la distribución. En el cuadro es posible identificar tres bloques de países. El primer bloque corresponde a países con baja desigualdad y en él se ubica

\footnotetext{
5 Como la no respuesta y el trabajo familiar no remunerado se concentran en las actividades agrícolas y las zonas rurales, estos dominios estarían también subrepresentados.
} 
Costa Rica. Este país presenta la distribución con menor desigualdad, cualquiera sea la medida de desigualdad que se utilice o el subgrupo de trabajadores que se analice. ${ }^{6} \mathrm{El}$ segundo bloque corresponde a países con desigualdad moderada y en él se ubica El Salvador. El Salvador muestra una desigualdad mayor que la de Costa Rica pero menor que la del resto de los países de la subregión. El último bloque corresponde a países con alta desigualdad y en él se ubican Guatemala, Honduras y Nicaragua. Guatemala aparece como el país con la mayor desigualdad cuando se usa el coeficiente de Gini o la varianza del logaritmo del ingreso, en tanto que Nicaragua muestra el mayor valor en el índice de Theil.

Cabe señalar que cuando se examina la desigualdad de los ingresos del trabajo, estos resultados dependen de los individuos que están trabajando. En otras palabras, dependen de las tasas de desempleo y de la elección de participar en la fuerza de trabajo. Como se ha señalado, no se producen aumentos significativos en el desempleo en el período bajo estudio y sólo Nicaragua presenta una reducción importante que puede asociarse con la evolución de la desigualdad que se examina más adelante. Las tasas de participación de los hombres no muestran mayores cambios en este lapso, en tanto que aumenta la participación de las mujeres en todos los países. Como se ve más adelante, este fenómeno ayuda a explicar el aumento en la dispersión de las horas trabajadas y su impacto en el incremento de la desigualdad.

\section{Las fuentes de la desigualdad: la descompo- sición de Fields}

Para examinar las fuentes de la desigualdad de los ingresos del trabajo se usa la técnica desarrollada por Fields ${ }^{7}$ y extendida por Yun (2002), que permite descomponer la desigualdad de las remuneraciones mensuales en componentes atribuidos a cambios asociados con características personales y del puesto de trabajo en que se desempeñan los trabajadores. Esta técnica de descomposición se basa en la estimación de ecuaciones de remuneración semilogarítmicas tradicionales

$$
\ln Y_{i t}=\Sigma_{j} B_{t j} * X_{i t j}+E_{i t}=\Sigma_{j} B_{t j} * Z_{i t j}
$$

\footnotetext{
${ }^{6}$ Estos subgrupos se presentan para verificar si la distinta presencia de trabajo asalariado y el distinto peso de las zonas rurales en los países pueden estar influyendo en los resultados.

${ }^{7}$ Véase Fields (2003) y Fields y Gyeongjoon (2000).
}

donde:

$\ln Y_{i t}$ es el logaritmo del ingreso laboral mensual, las $X_{i t j}$ son las variables $j$ asociadas con la persona $i$ en el año $t$ que pueden afectar las remuneraciones. Los coeficientes $B_{t j}$ miden los "precios" o premios salariales por cada variable $X$. Por ejemplo, el coeficiente sobre los años de educación mide el incremento de salario que un empleador tiene que pagar por un trabajador con un año más de educación. El residuo $E_{i t}$ es la parte de la variación en las remuneraciones entre los trabajadores, que no puede ser explicada por la variación entre las variables incluidas en la ecuación.

Se puede ilustrar la derivación de la descomposición de Fields utilizando la varianza del logaritmo de los ingresos como una medida de dispersión. Dada la ecuación de remuneración, la varianza del logaritmo de las remuneraciones puede escribirse así:

$$
\begin{gathered}
\operatorname{Var}\left(\ln Y_{i t}\right)=\operatorname{Cov}\left(\ln Y_{i t}, \ln Y_{i t}\right)= \\
\operatorname{Cov}\left(\Sigma_{j} B_{t j} * Z_{i t j}, \ln Y_{i t}\right)= \\
\sum_{j} \operatorname{Cov}\left(B_{t j} * Z_{i t}, \ln Y_{i t}\right)
\end{gathered}
$$

Dividiendo la ecuación [2] por la varianza del logaritmo de las remuneraciones,

$$
\frac{1=\Sigma_{j} \operatorname{Cov}\left(B_{t j} * Z_{i t j}, \ln Y_{i t}\right)}{\operatorname{Var}\left(\ln Y_{i t}\right)}=\Sigma_{j} S_{j t}
$$

Los $S_{j t}$ miden la proporción de la varianza en el logaritmo de los ingresos que es explicada por cada variable $j$ en el país o año $t$. Shorrocks (1982) ha mostrado que si se puede describir el ingreso (o el logaritmo del ingreso) como la suma de distintos componentes, entonces los $S_{j t}$ miden la contribución de cada variable $j$ a la desigualdad para un número amplio de medidas de desigualdad (no sólo para la varianza), incluyendo el coeficiente de Gini y el índice de Theil. ${ }^{8}$

Si bien se pueden usar los $S_{j t}$ para medir la contribución de cada variable $j$ al nivel de desigualdad, para medir el impacto de cada variable en las diferencias de la desigualdad entre países o en el tiempo es necesario utilizar algo más que los $S_{j t}$. Esto es así debido a que la magnitud de las diferencias de la desigualdad entre países o en el tiempo (y a veces hasta la dirección del cambio) dependerá de la medida de

\footnotetext{
${ }^{8}$ La descomposición funciona sólo si las variables son completamente linealizables. Esto excluye la posibilidad de interacciones entre las variables del lado derecho de la ecuación.
} 
desigualdad utilizada. Entonces, para medir la contribución de cada variable al cambio en la desigualdad, es necesario multiplicar los $S_{j t}$ en cada período o país $t$ por la medida de desigualdad correspondiente. Específicamente, si $I(t)$ es la medida de desigualdad en el período o país $t$, el cambio en la desigualdad entre períodos o países 1 y 2 puede ser escrito como:

$$
I(2)-I(1)=\Sigma_{j}\left\{I(2) * S_{j 2}-I(1) * S_{j 1}\right\}
$$

La ecuación [4] puede usarse para medir la contribución de cada variable al cambio en la desigualdad entre períodos o países.

Las variables utilizadas sobre las características del mercado de trabajo son: las horas trabajadas expresadas en logaritmo y un conjunto de variables binarias que representan el aporte o premio salarial de trabajar en el sector público (sector institucional), en las empresas formales o grandes (tamaño del establecimiento) y en las distintas ramas industriales (industria), donde la rama de minas y canteras es la de control. ${ }^{9}$ Las carac- terísticas de las personas incluyen variables asociadas con su capital humano, como años de educación formal y años de experiencia potencial, así como dos variables binarias que recogen el premio salarial que reciben los hombres con respecto a las mujeres (sexo) y la residencia en las zonas urbanas (zona). ${ }^{10}$

El cuadro 3 presenta los $S_{j t}$ [ecuación 3], o la proporción de la desigualdad en las remuneraciones que se explica por variables asociadas con características del mercado de trabajo y características personales en cada país. ${ }^{11}$ Los resultados muestran que en todos los países la educación es la fuente más importante de desigualdad cuantificada, pues explica entre el $18 \%$ y el $25 \%$ de la desigualdad total. Las diferencias de horas trabajadas entre los distintos trabajadores hacen aportes muy disímiles en los distintos países, aunque en general tienden a ocupar el segundo lugar como factor explicativo. Costa Rica es el país donde esas diferencias tienen mayor peso (18\%), seguido por Guatemala, donde originan un $11 \%$ de la desigualdad total y cerca de la mitad de la originada

CUADRO 3

Centromérica: Descomposición de Fields de la desigualdad

de los ingresos laborales, por país, a fines del decenio de 1990

(Para ocupados de 15 o más años con ingreso y horas trabajadas conocidas)

\begin{tabular}{lccccc}
\hline $\begin{array}{l}\text { Medida de desigualdad y grupo } \\
\text { de ocupados }\end{array}$ & Costa Rica & El Salvador & Guatemala & Honduras & Nicaragua \\
1998 & 1999 & 1999 & 1998 & 1999 & 1998 \\
\hline
\end{tabular}

Proporción de la desigualdad explicada

por cada característica $(\mathrm{Sj})$

Todas las características

Educación (años)

Sexo (hombre $=1$ )

Zona (urbano $=1$ )

Horas trabajadas (logaritmo)

Sector institucional (público $=1$ )

Tamaño establecimiento (6 o más $=1$ )

Experiencia (edad-educación-6)

Industria (conjunto variables binarias por rama)

Residuo

$\begin{array}{lrrrr}\mathbf{1 , 0 0} & \mathbf{1 , 0 0} & \mathbf{1 , 0 0} & \mathbf{1 , 0 0} & \mathbf{1 , 0 0} \\ 0,19 & 0,25 & 0,19 & 0,21 & 0,18 \\ 0,03 & 0,02 & 0,05 & 0,03 & 0,01 \\ 0,01 & 0,03 & 0,03 & 0,05 & 0,02 \\ 0,18 & 0,06 & 0,11 & 0,06 & 0,01 \\ 0,02 & 0,05 & 0,00 & 0,01 & -0,01 \\ 0,06 & 0,06 & 0,03 & 0,07 & 0,03 \\ 0,00 & 0,00 & 0,00 & -0,01 & 0,00 \\ 0,02 & 0,03 & 0,02 & 0,03 & 0,08 \\ 0,50 & 0,51 & 0,56 & 0,55 & 0,68\end{array}$

Fuente: Cálculos de los autores con base en las encuestas de hogares de los países y años respectivos.

\footnotetext{
${ }^{9}$ Como los países utilizan distintos clasificadores de ramas industriales, la información se ha homogeneizado para que corresponda con las nueve grandes divisiones de la Clasificación Industrial Internacional Uniforme de todas las actividades económicas (CIIU, Rev. 2).

${ }^{10}$ En la descomposición se sigue una relación lineal entre los años de educación y el logaritmo del ingreso. Como señala Contreras (2003), en Chile, entre otros países, existe evidencia que sugiere que esto puede no ser así. Para tomar en cuenta la posibilidad de que la relación no fuera lineal, se estimaron ecuaciones y las des-
}

composiciones, utilizando cuatro variables binarias para educación (primaria completa, secundaria incompleta, secundaria completa y educación superior). Los resultados obtenidos con esta especificación son muy similares y no modifican las conclusiones sobre el papel de la distribución y el rendimiento de la educación en la magnitud y evolución de la desigualdad y en las diferencias entre países. Por tal motivo se mantiene la especificación lineal propuesta inicialmente y cuyos resultados básicos se presentan en el apéndice $\mathrm{A}$.

11 Véase en el apéndice A las ecuaciones de remuneración. 
en Costa Rica; en Honduras (6\%) y El Salvador (6\%) su poder explicativo se reduce a cerca de la mitad del indicado para los países anteriores y en Nicaragua desempeña un papel marginal $(1 \%)$.

Las diferencias entre empresas grandes (formales) y pequeñas (informales) causan entre el $7 \%$ y el $6 \%$ de la desigualdad total en Honduras, Costa Rica y El Salvador. Las diferencias entre industrias explican $8 \%$ de esa desigualdad en Honduras. Individualmente, ninguna otra característica alcanza a explicar ni un 5\% de la desigualdad total en algún país. Esto significa que la desigualdad asociada con las características medidas llegan a explicar como máximo un 50\% de la desigualdad total (Costa Rica) y como mínimo un 32\% (Nicaragua), debiéndose el complemento al residuo de la ecuación de remuneración. La parte de la desigualdad que se debe al residuo resulta de la desigualdad entre personas con la misma educación, sexo, zona, horas trabajadas, sector institucional, tamaño del establecimiento, experiencia y rama industrial.

\section{IV}

\section{Las fuentes de los cambios en la desigualdad en el decenio de 1990}

El análisis de los cambios en la desigualdad durante la década de 1990 presenta mayores dificultades, pues sólo a fines de ese período comienzan a generalizarse las encuestas de hogares. En esta sección se usa la encuesta más cercana al inicio del decenio de 1990 disponible en cada país, con el fin de visualizar la evolución en dicho decenio. Las encuestas utilizadas son la de 1989 para Guatemala, de 1990 para Costa Rica y Honduras, de 1993 para Nicaragua y de 1995 para El Salvador. Los datos sobre los inicios de la década también corresponden a años cercanos al comienzo de los programas de ajuste estructural.

\section{Cambios en la desigualdad de la distribución de los ingresos laborales}

El cuadro 4 presenta los indicadores de desigualdad para cada país centroamericano, usando datos de principios

CUADRO 4

Centroamérica: Evolución de la desigualdad del ingreso laboral, por país, en el decenio de 1990

(De las personas ocupadas con ingresos y de 15 o más años ordenadas según su ingreso laboral)

\begin{tabular}{|c|c|c|c|c|c|}
\hline Indicador & Costa Rica & El Salvador & Guatemala & Honduras & Nicaragua \\
\hline \multicolumn{6}{|l|}{ Año de la encuesta } \\
\hline Cerca de 1990 & 1990 & 1995 & 1989 & 1990 & 1993 \\
\hline Cerca de 1999 & 1999 & 1999 & 1998 & 1999 & 1998 \\
\hline \multicolumn{6}{|c|}{ Coeficiente de Gini } \\
\hline Cerca de 1990 & 0,410 & 0,462 & 0,517 & 0,562 & 0,542 \\
\hline Cambio & 0,026 & 0,007 & 0,060 & $-0,019$ & 0,021 \\
\hline \multicolumn{6}{|l|}{ Índice de Theil } \\
\hline Cerca de 1990 & 0,319 & 0,447 & 0,563 & 0,759 & 0,560 \\
\hline Cerca de 1999 & 0,347 & 0,412 & 0,701 & 0,583 & 0,705 \\
\hline Cambio & 0,027 & $-0,035$ & 0,139 & $-0,176$ & 0,145 \\
\hline \multicolumn{6}{|c|}{ Varianza del logaritmo del ingreso } \\
\hline Cerca de 1999 & 0,775 & 0,779 & 1,436 & 1,203 & 1,039 \\
\hline Cambio & 0,072 & 0,093 & 0,411 & 0,174 & $-0,132$ \\
\hline
\end{tabular}

Fuente: Cálculos de los autores con base en las encuestas de hogares de los países y años respectivos. 
GRAFICO 3

Centroamérica: Cambio en los indicadores de la desigualdad del ingreso laboral en el decenio de 1990

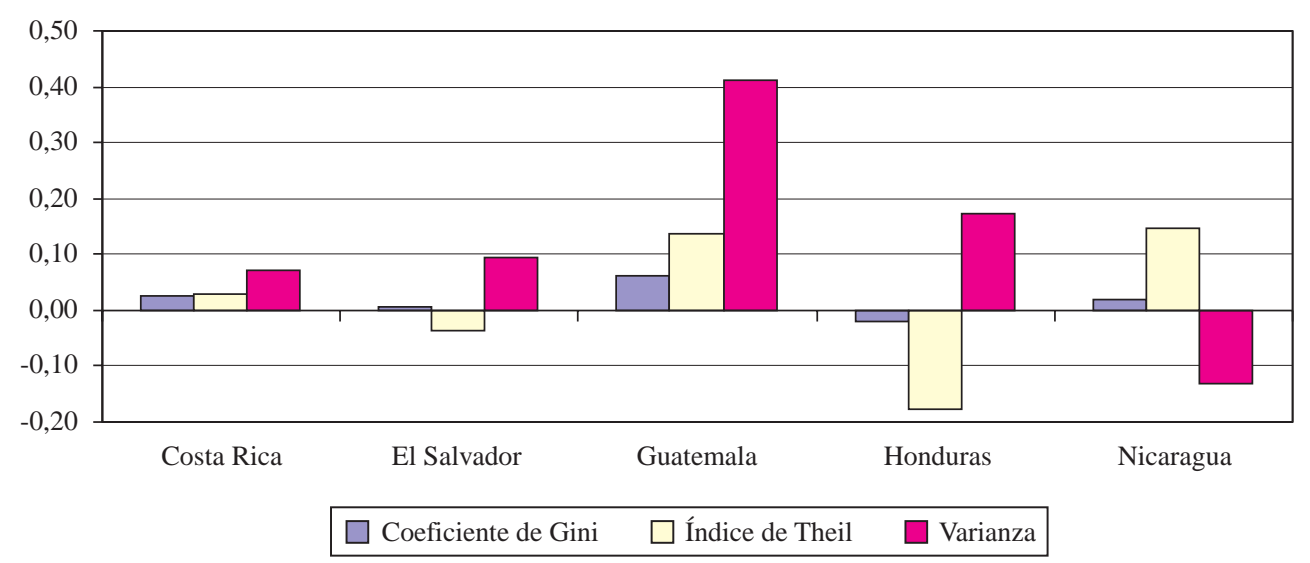

Fuente: Cuadro 4.

de la década de 1990, y los compara con los indicadores de finales del decenio ya analizados en las secciones anteriores.

Los datos del cuadro 4 indican que la desigualdad en la distribución de los ingresos del trabajo se habría incrementado sin ambigüedad, pues los tres indicadores lo corroboran en Costa Rica y Guatemala. En este grupo Guatemala mostraría el mayor aumento de la desigualdad. En Nicaragua y Honduras la desigualdad se habría incrementado o habría disminuido según el indicador que se utilice (gráfico 3). La varianza del logaritmo del ingreso, que es más sensible a los cambios en la parte baja de la distribución, cae en Nicaragua, mientras aumentan el coeficiente de Gini, que es más sensible a los cambios en la parte media de la distribución, y el índice de Theil, más sensible a cambios en la parte superior de la distribución. Esto sugiere que los ingresos laborales de los trabajadores en la base de la distribución crecen (crecen más o se reducen menos) en relación con los ingresos de los trabajadores en el medio de la distribución, mientras que las remuneraciones del grupo medio no aumentan más rápidamente que las de los trabajadores en la parte superior de la distribución. La reducción marcada del desempleo, que se asocia con mayores oportunidades de empleo para los trabajadores menos calificados, puede explicar esta evolución. Otra posibilidad es que haya un problema con los datos de Nicaragua, en particular, que existan datos muy fuera de rango en la parte baja de la distribución que podrían causar la caída en la varianza del logaritmo de los ingresos. Para examinar esta posibilidad se elimina el $1 \%$ de los más pobres y se reestima el cambio en la varianza de dicho logaritmo. Cuando se hace esto, la varianza del logaritmo de los ingresos en Nicaragua aumenta entre 1993 y 1998, lo que sugiere que el descenso observado en la varianza del logaritmo de los ingresos puede deberse a problemas con los datos. En Honduras, la varianza del logaritmo de los ingresos se acrecentó, mientras que el coeficiente de Gini y el índice de Theil se redujeron. ${ }^{12}$

La evolución descrita no modifica el ordenamiento de los países en los tres bloques de desigualdad mencionados. Costa Rica se mantiene en el bloque de menor desigualdad y El Salvador en el de desigualdad intermedia. Guatemala, Honduras y Nicaragua siguen siendo los países con mayor desigualdad en el decenio de 1990, pese a que según el año y el indicador se ordenan en forma diferente. Aplicando el coeficiente de Gini, Honduras era el país más desigual cerca de 1990 y Guatemala al final del decenio. Con el índice de Theil, Honduras era el más desigual en 1990 y Nicaragua lo era a finales de la década. Finalmente, con la varianza del logaritmo del ingreso, alrededor de 1990

\footnotetext{
12 Este resultado no se debe a que haya datos muy fuera de rango, pues en Honduras se mantuvo aunque eliminamos los $1 \%$ más pobres en la distribución en cada año. Cabe señalar que las muestras superan el $1 \%$ de la población en Costa Rica y El Salvador, y son más pequeñas para los demás países (Székely y Hilgert, 1999a). Para estos autores, las muestras pequeñas en Honduras, Guatemala y Nicaragua se traducen en mayores errores estadísticos.
} 
(Para ocupados de 15 o más años con ingreso y horas trabajadas conocidas)

\begin{tabular}{lccccc}
\hline Variables & Costa Rica & El Salvador & Guatemala & Honduras & Nicaragua \\
& 1990 & 1995 & 1989 & 1990 & 1993 \\
\hline
\end{tabular}

Proporción de la desigualdad explicada

por cada característica $(\mathrm{Sj})$

Todas las características

Educación (años)

Sexo $($ hombre $=1)$

Zona (urbano $=1$ )

Horas trabajadas (logaritmo)

Sector institucional (público $=1$ )

Tamaño establecimiento (6 o más $=1$ )

Experiencia (edad-educación-6)

Industria (conjunto variables binarias por rama)

Residuo

$\begin{array}{rrrrr}\mathbf{1 , 0 0} & \mathbf{1 , 0 0} & \mathbf{1 , 0 0} & \mathbf{1 , 0 0} & \mathbf{1 , 0 0} \\ 0,19 & 0,19 & 0,21 & 0,24 & 0,18 \\ 0,02 & 0,03 & 0,01 & 0,04 & 0,00 \\ 0,01 & 0,05 & 0,03 & 0,04 & 0,06 \\ 0,13 & 0,06 & 0,03 & 0,04 & 0,02 \\ 0,04 & 0,02 & 0,02 & -0,01 \\ 0,06 & 0,03 & 0,09 & 0,07 & 0,06 \\ 0,01 & 0,04 & -0,02 & -0,01 & -0,01 \\ 0,03 & 0,00 & 0,09 & 0,01 & 0,09 \\ 0,52 & 0,06 & 0,56 & 0,55 & 0,61\end{array}$

Fuente: Cálculos de los autores con base en las encuestas de hogares de los países y años respectivos.

Nicaragua se tornaba más desigual y a finales de la década lo hacía Guatemala.

En resumen, los datos no muestran un patrón uniforme en la evolución de la distribución del ingreso laboral en la década de 1990; dicho patrón depende del indicador que se utilice. Solamente Costa Rica y Guatemala presentan un deterioro claro en la distribución del ingreso. Pero bajo estos cambios brutos hay fenómenos comunes a los mercados de trabajo de todos los países. Estos fenómenos pueden identificarse mediante el análisis de las fuentes de los cambios en la desigualdad del ingreso laboral.

\section{Las fuentes de la desigualdad del ingreso la- boral a inicios de la década de 1990}

El cuadro 5 es una réplica del cuadro 3, utilizando datos cercanos a 1990. Al inicio de la década de 1990, la educación se mantiene como la variable que más incide en la desigualdad de los ingresos laborales. Sólo en Costa Rica las horas trabajadas se mantienen como la segunda en importancia de las fuentes de desigualdad, en tanto que en Guatemala y Nicaragua son más significativas en conjunto las brechas salariales entre las industrias y, en Honduras, la brecha salarial por tamaño. Esta última variable también tiene importancia como factor de desigualdad en el resto de los países de la subregión, mientras que el residuo, o la parte que las variables incorporadas no explican, muestra un peso similar por país al que se observa al final de decenio.
Se ha señalado que para medir la contribución de cada variable al cambio en la desigualdad, es necesario multiplicar los $S_{j t}$ en cada período o país $t$ por la medida de desigualdad correspondiente [ecuación 4]. En el cuadro 6 se muestra la contribución de cada variable al cambio en una de las medidas de la desigualdad: la varianza del logaritmo del ingreso del trabajo. ${ }^{13}$ Como indica el cuadro, en todos los países excepto Nicaragua tres fenómenos impulsaron el incremento de la desigualdad del ingreso laboral en el decenio de 1990: los cambios relacionados con la educación, las horas trabajadas y el residuo. En Guatemala, los cambios relacionados con el sexo del trabajador y con la experiencia también apoyan el aumento de la desigualdad. Por otro lado, los cambios que se asocian al tamaño de la empresa y a la rama industrial en que se desempeña el trabajador promueven una reducción en la desigualdad en Guatemala y Nicaragua. Las otras variables tienen impactos pequeños sobre los cambios en la desigualdad.

\section{La descomposición de Yun: distinguir los efec- tos de diferencias en remuneraciones y distri- buciones de las características personales}

Cada variable puede contribuir a las diferencias en la desigualdad total entre países o en el tiempo, porque

\footnotetext{
${ }^{13}$ Recuérdese que cuando se utiliza la varianza del logaritmo del ingreso como indicador de desigualdad, ésta aumenta en todos los países, salvo Nicaragua.
} 
Centroamérica: Contribución de cada variable al cambio en la varianza del logaritmo (Sj*VarLogY)

(Para ocupados de 15 o más años con ingreso y horas trabajadas conocidas)

\begin{tabular}{|c|c|c|c|c|c|}
\hline Variables & $\begin{array}{c}\text { Costa Rica } \\
1990 / 1999\end{array}$ & $\begin{array}{c}\text { El Salvador } \\
1995 / 1999\end{array}$ & $\begin{array}{c}\text { Guatemala } \\
1989 / 1998\end{array}$ & $\begin{array}{c}\text { Honduras } \\
1990 / 1999\end{array}$ & $\begin{array}{l}\text { Nicaragua } \\
1993 / 1998\end{array}$ \\
\hline Cambio en la varianza del logaritmo & 0,08 & $\mathbf{0 , 1 0}$ & 0,41 & $\mathbf{0 , 1 7}$ & $-0,13$ \\
\hline Educación (años) & 0,02 & 0,06 & 0,07 & 0,01 & $-0,02$ \\
\hline Sexo $($ hombre $=1)$ & 0,00 & $-0,01$ & 0,06 & 0,00 & 0,01 \\
\hline Zona $($ urbano $=1)$ & 0,00 & $-0,01$ & 0,02 & 0,02 & $-0,04$ \\
\hline Horas trabajadas (logaritmo) & 0,05 & 0,01 & 0,13 & 0,04 & $-0,02$ \\
\hline Sector institucional (público = 1) & $-0,01$ & 0,02 & $-0,01$ & $-0,01$ & 0,00 \\
\hline Tamaño establecimiento (6 o más $=1)$ & 0,00 & 0,02 & $-0,04$ & 0,01 & $-0,04$ \\
\hline Experiencia (edad-educación-6) & 0,00 & 0,00 & 0,03 & $-0,01$ & 0,01 \\
\hline Industria (conjunto variables binarias por rama) & 0,00 & $-0,02$ & $-0,06$ & 0,02 & $-0,03$ \\
\hline Residuo & 0,03 & 0,02 & 0,23 & 0,10 & $-0,02$ \\
\hline
\end{tabular}

Fuente: Cálculos de los autores con base en las encuestas de hogares de los países y años respectivos.

los precios (coeficientes $B_{j}$ ) de esas características difieren entre los países o en el tiempo, o porque la dispersión de esas características (cambios en la dispersión de $Z_{j}$ ) difieren entre los países o en el tiempo. Por ejemplo, el cuadro 6 muestra que en el decenio de 1990 los cambios relacionados con la educación contribuyeron a acentuar la desigualdad en la mayoría de los países centroamericanos. Pero esto podría ser porque se elevó el precio de la educación (el coeficiente sobre la variable años de educación), o bien, porque la distribución de niveles de educación entre trabajadores es más desigual. Resulta muy útil entonces poder distinguir entre cambios en la desigualdad causados por variaciones en los precios-coeficientes y cambios causados por variaciones en la distribución de cada $Z_{j}$. Yun (2002) deriva una extensión de la descomposición de Fields para la varianza del logaritmo de las remuneraciones que permite esta separación. Con tal fin - siguiendo la lógica de Juhn, Murphy y Pierce (1993 - construye una distribución “auxiliar", usando los $B s$ del período o país 2 y las $Z s$ del período o país 1:

$$
\ln Y_{i, \text { aux }}=\Sigma_{j} B_{2 j} * X_{i l j}+E_{i l}=\Sigma_{j} B_{2 j} * Z_{i l j}
$$

El cambio en la varianza del logaritmo de las remuneraciones puede escribirse como

$$
\begin{gathered}
\operatorname{Var}\left(\ln Y_{2}\right)-\operatorname{Var}\left(\ln Y_{1}\right)=\left[\operatorname{Var}\left(\ln Y_{\text {aux }}\right)-\operatorname{Var}\left(\ln Y_{1}\right)\right]+ \\
{\left[\operatorname{Var}\left(\ln Y_{2}\right)-\operatorname{Var}\left(\ln Y_{\text {aux }}\right)\right]=\Sigma_{j}\left[S_{j a u x^{*}} \operatorname{Var}\left(\ln Y_{\text {aux }}\right)-\right.} \\
\left.S_{j 1^{*}} \operatorname{Var}\left(\ln Y_{1}\right)\right]+\left[S_{j 2^{*}} \operatorname{Var}\left(\ln Y_{2}\right)-\right. \\
\left.S_{j a u *^{*}} \operatorname{Var}\left(\ln Y_{\text {aux }}\right)\right]
\end{gathered}
$$

$$
\begin{gathered}
=\Sigma_{j}\left[B_{2 j} * D E\left(Z_{i l j}\right) * \operatorname{Corr}\left(Z_{i l j,} \ln Y_{a u x}\right) * D E\left(\ln Y_{a u x}\right)-\right. \\
\left.B_{l j}{ }^{*} D E\left(Z_{i l j}\right) * \operatorname{Corr}\left(Z_{i l j,} \ln Y_{a l}\right) * D E\left(\ln Y_{a l}\right)\right]+ \\
\sum_{j}\left[B_{2 j} * D E\left(Z_{i 2 j}\right) * \operatorname{Corr}\left(Z_{i j j,}, \ln Y_{2}\right) * D E\left(\ln Y_{2}\right)-\right. \\
\left.B_{2 j}{ }^{*} D E\left(Z_{i l j}\right) * \operatorname{Corr}\left(Z_{i l j,} \ln Y_{a u x}\right) * D E\left(\ln Y_{a u x}\right)\right]
\end{gathered}
$$

donde la primera línea de la ecuación [7] muestra la contribución a la diferencia (entre períodos o países) en la varianza del logaritmo del ingreso debido a cambios o diferencias en cada uno de los coeficientes, mientras que la segunda línea muestra la contribución de los cambios o diferencias en la varianza de cada $Z_{j^{\prime}}{ }^{14}$

El cuadro 7 y el gráfico 4 presentan la descomposición de Yun de los cambios en la desigualdad de los ingresos del trabajo (medido con la varianza del logaritmo del ingreso laboral) en cada país de Centroamérica durante la década de $1990 .{ }^{15}$ Un primer resultado que cabe destacar es que en todos los países, salvo Nicaragua, un aumento de la desigualdad en el número de horas trabajadas entre los trabajadores (efecto distribución) promueve un incremento en la desigualdad de las remuneraciones. El efecto de horas trabajadas es más grande en Guatemala y Costa Rica que en los demás países centroamericanos.

\footnotetext{
${ }^{14}$ Un posible problema de las ecuaciones de remuneración es el sesgo de selección, y la corrección tradicional es la técnica de Heckman. Las ecuaciones de remuneración no han sido estimadas con la corrección de Heckman, porque no es posible integrar esa técnica con la descomposición propuesta por Yun aquí seguida.

${ }^{15}$ Véase en Gindling y Trejos (2004) una aplicación de estas técnicas a Costa Rica, con un horizonte temporal mayor.
} 
CUADRO 7

Centroamérica: Descomposición de Yun de las diferencias en la desigualdad de los ingresos laborales

(Para ocupados de 15 o más años con ingreso y horas trabajadas conocidas)

\begin{tabular}{|c|c|c|c|c|c|}
\hline Variables & $\begin{array}{c}\text { Costa Rica } \\
1990 / 1999\end{array}$ & $\begin{array}{c}\text { El Salvador } \\
1995 / 1999\end{array}$ & $\begin{array}{c}\text { Guatemala } \\
1989 / 1998\end{array}$ & $\begin{array}{l}\text { Honduras } \\
1990 / 1999\end{array}$ & $\begin{array}{l}\text { Nicaragua } \\
1993 / 1998\end{array}$ \\
\hline
\end{tabular}

Contribución de los cambios en los coeficientes de cada variable

Efecto precio total

Educación (años)

Sexo $($ hombre $=1)$

Zona (urbano $=1$ )

Horas trabajadas (logaritmo)

Sector institucional (público $=1$ )

Tamaño establecimiento $(6$ o más $=1)$

Experiencia (edad-educación-6)

Industria (conjunto varias binarias por rama)

$\begin{array}{rr}\mathbf{- 0 , 0 2} & \mathbf{0 , 0 4} \\ 0,00 & 0,05 \\ 0,00 & -0,01 \\ 0,00 & -0,01 \\ 0,00 & 0,00 \\ 0,00 & 0,02 \\ 0,00 & 0,01 \\ -0,01 & 0,00 \\ -0,01 & -0,02\end{array}$

Contribución de los cambios en la distribución de cada variable

\section{Efecto distribución total}

Educación (años)

Sexo (hombre $=1$ )

Zona (urbano = 1)

Horas trabajadas (logaritmo)

Sector institucional (público $=1$ )

Tamaño establecimiento $(6$ o más $=1)$

Experiencia (edad-educación-6)

Industria (conjunto varias binarias por rama)

0,07

0,01

0,01

0,00

0,05

0,00

0,00

0,00

0,00
0,03

0,01

0,00

0,00

0,01

0,00

0,01

0,00

0,00

$\mathbf{0 , 0 7}$
0,04
0,05
0,01
0,04
$-0,01$
$-0,04$
0,03
$-0,06$

$\mathbf{0 , 0 4}$

$-0,02$

0,00

0,02

0,02

$-0,01$

0,01

$-0,01$

0,03

$-0,14$

$-0,03$

0,02

$-0,04$

$-0,01$

0,00

$-0,04$

0,01

$-0,04$

Fuente: Cálculos de los autores con base en las encuestas de hogares de los países y años respectivos.

Centroamérica: Descomposición de Yun de las diferencias en la desigualdad de los ingresos laborales

Contribución de los cambios en la distribución de cada variable

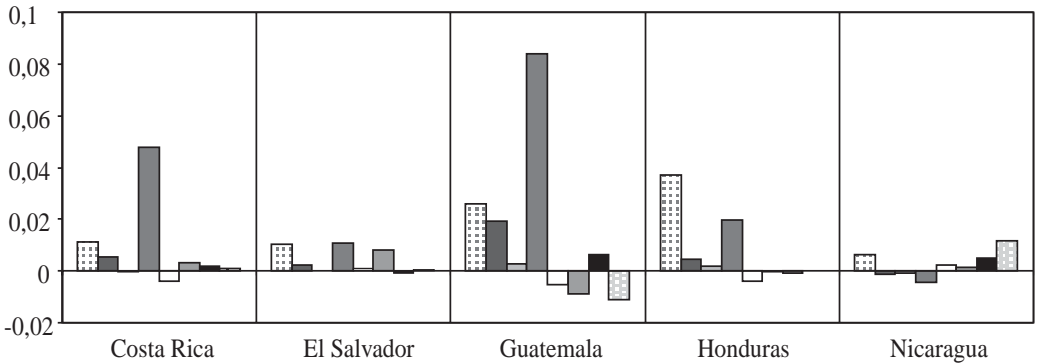

Contribución de los cambios en los coeficientes de cada variable

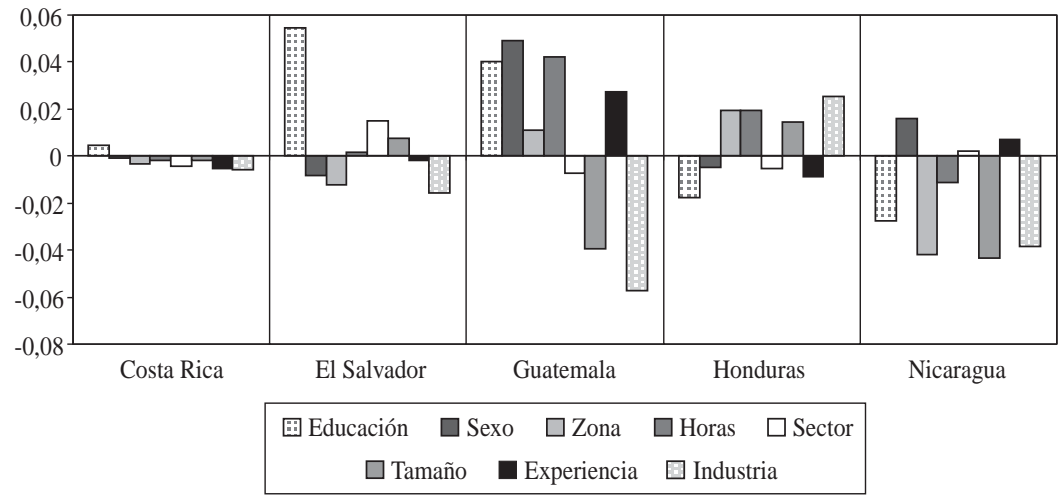

Fuente: Cuadro 7. 
La desigualdad en las horas trabajadas aumentó porque se redujo la proporción de trabajadores con jornadas completas en cada país y se elevó la proporción de aquellos con jornada parcial y con sobrejornada $^{16}$ (cuadro 8). En parte, la baja en la proporción de trabajadores con jornada completa se debió al incremento de la participación de mujeres en la fuerza de trabajo, ya que estas trabajan jornada parcial con mayor frecuencia que los hombres. En parte, el incremento de la desigualdad en las horas trabajadas derivó del aumento de la proporción de trabajadores en empresas pequeñas, los que tienen más probabilidades de trabajar jornadas parciales y sobrejornadas. La desigualdad en las horas trabajadas se acentuó también porque disminuyó la proporción de trabajadores en el sector público, donde predomina la jornada completa. Tal disminución es el resultado de las reformas económicas emprendidas en la subregión. Asimismo, los cambios en las horas trabajadas dentro de cada sector, sexo e industria contribuyeron a incrementar la proporción de trabajadores con jornada parcial y sobrejornada.

El aumento del trabajo con sobrejornada ocurrió entre trabajadores de las empresas privadas de mayor tamaño, mientras que en la mayoría de los países la proporción de trabajadores con sobrejornada bajó en el sector público, y en algunos países también en las empresas pequeñas. La mayor proporción de trabajadores con sobrejornada en las empresas privadas grandes puede ser un resultado de las reformas económicas, que crearon la necesidad de mejorar la productividad de sus trabajadores y la competitividad de sus empresas.

En el otro extremo, el incremento de los trabajadores con jornada parcial se dio principalmente en las empresas pequeñas (el sector informal). En los dos países donde la desigualdad en las horas trabajadas aumentó más, Costa Rica y Guatemala, se elevó considerablemente la proporción de mujeres ocupadas en las empresas pequeñas, que tienden a trabajar jornadas parciales. Esta feminización e informalización de la fuerza de trabajo en Centroamérica se describe en Trejos (2002). ${ }^{17}$

Cabe destacar aquí otros resultados (cuadro7): en todos los países centroamericanos la desigualdad en la distribución de la educación entre los trabajadores apoya una mayor desigualdad en la distribución de los

\footnotetext{
16 Por "sobrejornada" entendemos una jornada de trabajo superior a la jornada legal o normal, que en Centroamérica corresponde a una semana de trabajo de hasta 48 horas. En este sentido, la sobrejornada corresponde a una semana de trabajo de 49 horas o más.

${ }^{17} \mathrm{El}$ incremento en la proporción de trabajadores con jornada parcial ocurrió en las industrias que producen bienes no transables. En todos los países las ramas industriales que exhiben mayores au-
}

ingresos del trabajo. Además, en Guatemala y Nicaragua se observa un aumento en la brecha salarial en perjuicio de las mujeres, que apoya el aumento de la desigualdad (en Guatemala) o neutraliza parte de su reducción (en Nicaragua). En Costa Rica, El Salvador y Honduras la brecha salarial entre mujeres y hombres se estancó en el período 1990-1999.

Por otra parte, en ese período las brechas salariales entre trabajadores de empresas grandes y pequeñas se redujeron en Guatemala y Nicaragua, no cambiaron en Costa Rica y aumentaron en Honduras y El Salvador. Esto significa que mientras las diferencias de remuneraciones entre las empresas grandes y pequeñas en Guatemala y Nicaragua fueron una importante fuente de desigualdad a comienzos del decenio de 1990, hacia finales del decenio ya no lo eran.

Por último, y salvo en Honduras, las diferencias salariales entre las industrias se redujeron en la década de 1990 y contribuyeron a reducir la desigualdad. Además, en todos los países de la subregión los cambios en la composición de los trabajadores dentro de las distintas industrias tienen un impacto muy pequeño en los cambios observados en la desigualdad de la distribución de los ingresos laborales. Se ha sugerido que la liberalización comercial afectará la desigualdad de las remuneraciones mediante su efecto en la composición del empleo industrial y los cambios en los diferenciales salariales entre industrias. ${ }^{18}$ Estos resultados sugieren que tal efecto no explica de manera importante los cambios en la desigualdad de los ingresos laborales centroamericanos en la década de 1990.

Por otra parte, estos resultados son consistentes con los hallazgos de otros estudios, ${ }^{19}$ que con una metodología de microsimulaciones han encontrado que en la mayoría de los países las reformas económicas han estado acompañadas por una mayor desigualdad de los ingresos laborales, debido a una insuficiente creación de empleo moderno que expande el empleo informal (aumenta la dispersión de las horas trabajadas) y a un aumento del salario relativo de los más calificados (eleva el premio por años de educación).

mentos de trabajadores con jornada parcial son el comercio, la construcción y los servicios. Sólo en Nicaragua se incrementó la proporción de tales trabajadores en las industrias de bienes transables: la manufactura o la agricultura. Esto último implica que el aumento de la proporción de trabajadores con jornada parcial no se debe a subcontrataciones externas efectuadas por las empresas exportadoras grandes a empresas pequeñas o trabajadores en el hogar.

18 Véase Autor y Katz (1999), Katz y Murphy (1992), Koujianou y Pavcnik (2001) y Robertson (1999).

${ }^{19}$ Como los contenidos en Ganuza, Paes de Barros y otros ( 2001). 
CUADRO 8

Centroamérica: Cambios en la proporción de todos los trabajadores por sexo, sector y tipo de jornada

(Para ocupados de 15 o más años con ingreso y horas trabajadas conocidas)

Cambio en la proporción de trabajadores

Costa Rica
$1990 / 1999$

El Salvador
$1995 / 1999$

Guatemala
$1989 / 1998$

Honduras

Nicaragua

\section{A. Por tipo de jornada}

Jornada parcial (39 horas o menos)

Jornada completa (40 a 48 horas)

Sobrejornada (49 horas y más)

1,89
$-6,75$
4,86

$-3,26$
3,27

4,97
$-5,67$
0,70

15,95
$-16,47$
0,51
1990/1999 1993/1998

\section{B. Por sexo}

Hombres

Mujeres

5,46
$-0,40$
$-5,02$

$-4,84$
4,84

$-10,06$

10,06

5,72
$-7,28$

$-7,28$

0,24

C. Por Sector

Privado pequeño

Privado grande

Público

$-0,40$
$-5,02$

$$
\begin{array}{r}
3,40 \\
-2,86 \\
-0,54
\end{array}
$$
4,20
$-0,98$
$-3,23$

1,56

$-3,25$

D. Por sexo y tipo de jornada

Hombres, jornada parcial

Mujeres, jornada parcial

$-0,58$
2,48
3,29
1,57

2,78
2,18
$-2,24$
2,94

5,65
10,30
0,01
0,50

$$
-7,52
$$

7,52

Hombres, sobrejornada

Mujeres, sobrejornada

$\begin{array}{rrr}2,48 & 3,90 & 13,80 \\ 0,11 & 0,98 & 1,94 \\ -0,69 & 0,09 & 0,22 \\ & & \\ 3,71 & 0,99 & -1,83 \\ 1,46 & -0,01 & 2,58 \\ -0,30 & -0,28 & -0,24\end{array}$

$\begin{array}{rr}-0,32 & -0,17 \\ 2,29 & 7,82 \\ -1,94 & -7,66\end{array}$

E. Por sector y tipo de jornada

Privado pequeño, jornada parcial

Privado grande, jornada parcial

Público, jornada parcial

Privado pequeño, sobrejornada

Privado grande, sobrejornada

Publico, sobrejornada

$-0,30$

$-0,01$
$-0,28$

Fuente: Cálculos de los autores con base en las encuestas de hogares de los países y años respectivos.

\section{V \\ Fuentes de las diferencias en la desigualdad del ingreso laboral entre los países de Centroamérica}

En esta sección se comparan las fuentes de la desigualdad del ingreso laboral en Costa Rica con las fuentes de la desigualdad de los ingresos laborales en los demás países centroamericanos. Como Costa Rica tiene la distribución relativamente más igualitaria de la región, es conveniente utilizar este país como punto de referencia. El cuadro 9 presenta los resultados de la descomposición de Yun de la diferencia entre Costa Rica y los otros países de la subregión, en la desigual- dad medida por la varianza del logaritmo del ingreso laboral. El primer bloque del cuadro muestra la contribución de cada variable a esa diferencia observada, mientras que los otros dos bloques presentan el aporte separado de los precios y de la distribución de cada variable a la diferencia del indicador de desigualdad entre Costa Rica y cada uno de los restantes países de Centroamérica. En el cuadro, un número negativo indica que esta variable o fenómeno contribuye a 
Centroamérica: Descomposición de Yun de las diferencias en la desigualdad de los ingresos laborales con relación a Costa Rica, 1999

(Para ocupados de 15 o más años con ingreso y horas trabajadas conocidas)

\begin{tabular}{lcccc}
\hline Variables & El Salvador & Guatemala & Honduras & Nicaragua \\
& 1999 & 1998 & 1999 & 1998
\end{tabular}

Contribución de cada variable a las

diferencias en la Var Ln y

Diferencia en la varianza del logaritmo del ingreso

Explicada por las variables utilizadas

Educación (años)

Sexo $($ hombre $=1)$

Zona (urbano $=1$ )

Horas trabajadas (logaritmo)

Sector institucional (público $=1$ )

Tamaño establecimiento $(6$ o más $=1)$

Experiencia (edad-educación-6)

Industria (conjunto variables binarias por rama)

Residuo

Contribución de los cambios en los

coeficientes de cada variable

Efecto precio total

Educación (años)

Sexo (hombre $=1)$

Zona (urbano $=1)$

Horas trabajadas (logaritmo)

Sector institucional (público $=1$ )

Tamaño establecimiento ( 6 o más $=1)$

Experiencia (edad-educación-6)

Industria (conjunto variables binarias por rama)

Contribución de los cambios en la

distribución de cada variable

Efecto distribución total

Educación (años)

Sexo $($ hombre $=1)$

Zona (urbano $=1$ )

Horas trabajadas (logaritmo)

Sector institucional (público $=1$ )

Tamaño establecimiento $(6$ o más $=1$ )

Experiencia (edad-educación-6)

Industria (conjunto variables binarias por rama)

$\begin{array}{rrrr}\mathbf{0 , 0 1} & \mathbf{0 , 6 6} & \mathbf{0 , 4 3} & \mathbf{0 , 2 7} \\ \mathbf{- 0 , 0 1} & \mathbf{0 , 2 5} & \mathbf{0 , 1 5} & \mathbf{- 0 , 0 5} \\ 0,04 & 0,13 & 0,10 & 0,04 \\ -0,01 & 0,05 & 0,02 & -0,01 \\ 0,02 & 0,04 & 0,06 & 0,02 \\ -0,09 & 0,02 & -0,06 & -0,13 \\ 0,00 & -0,01 & -0,01 & -0,02 \\ 0,00 & 0,00 & 0,04 & -0,02 \\ -0,01 & 0,00 & -0,02 & 0,00 \\ 0,04 & 0,01 & 0,02 & 0,07 \\ \mathbf{0 , 0 1} & \mathbf{0 , 4 2} & \mathbf{0 , 2 8} & \mathbf{0 , 3 2}\end{array}$

$\begin{array}{rrrr}\mathbf{0 , 0 0} & \mathbf{0 , 2 2} & \mathbf{0 , 1 8} & \mathbf{- 0 , 0 1} \\ -0,02 & 0,10 & 0,08 & 0,01 \\ 0,00 & 0,04 & 0,02 & 0,00 \\ 0,02 & 0,04 & 0,06 & 0,02 \\ -0,03 & 0,01 & -0,04 & -0,09 \\ 0,02 & 0,00 & 0,00 & -0,02 \\ 0,00 & 0,01 & 0,05 & -0,01 \\ 0,01 & 0,02 & -0,01 & 0,01 \\ 0,02 & 0,02 & 0,02 & 0,07\end{array}$

Fuente: Cálculos de los autores con base en las encuestas de hogares de los países y años respectivos.

bajar la desigualdad en ese país en comparación con Costa Rica, mientras que un número positivo implica que esta variable o fenómeno contribuye a incrementar la desigualdad en ese país en comparación con Costa Rica.

Tres elementos importantes explican por qué las remuneraciones están distribuidas más equitativamente en Costa Rica que en cualquier otro país centroamericano: la educación, la zona de residencia y las brechas salariales por rama industrial.

\section{La educación}

Las diferencias en los niveles de educación entre los trabajadores causan menor desigualdad en Costa Rica que en los demás países de Centroamérica por dos factores: en primer lugar, la educación aparece mejor distribuida en Costa Rica. Esto se observa en el efecto distribución de la variable educación (cuadro 9) y se puede corroborar con los valores de la desviación estándar de esa misma variable que se presentan en el 
apéndice A, donde se resumen los estadísticos básicos de las ecuaciones de remuneración.

Este resultado surge porque la proporción de trabajadores con muy poca educación es más alta en los otros países centroamericanos que en Costa Rica. El porcentaje de trabajadores que no han completado la enseñanza primaria es de $20 \%$ en Costa Rica, $40 \%$ en El Salvador, $47 \%$ en Honduras, $48 \%$ en Nicaragua y $61 \%$ en Guatemala. En lo que toca a la educación superior, $16 \%$ de los trabajadores costarricenses de la muestra tiene algún grado de educación universitaria, porcentaje que contrasta con el $12 \%$ en El Salvador, $8 \%$ en Nicaragua y $5 \%$ en Honduras y Guatemala.

Otro factor del papel de la educación como diferenciador de las distribuciones de los ingresos del trabajo entre Costa Rica y el resto de la subregión, es el relativo al rendimiento de un año adicional de enseñanza. Este rendimiento es menor en Costa Rica que en el resto de la subregión salvo en El Salvador (cuadro 9 y apéndice A) y alcanza los mayores valores en los países con más bajo nivel educativo promedio (Guatemala y Honduras). En El Salvador, el efecto precio de la educación reduce la desigualdad en relación con Costa Rica, pero se ve contrarrestado con creces por una distribución más desigual de la educación, de modo que el efecto total de la educación en El Salvador contribuye a incrementar la desigualdad con referencia a Costa Rica.

\section{La zona de residencia}

Este segundo elemento contribuye a la mayor desigualdad en el resto de los países centroamericanos, comparados con Costa Rica, ya que en esos países el premio salarial que reciben los trabajadores urbanos es más alto que el que reciben los trabajadores rurales. Las diferencias cuantificadas en la proporción de trabajadores urbanos (efecto distribución) entre los países no desempeñan papel alguno en las diferencias de desigualdad entre Costa Rica y los otros países, pese a que en Costa Rica la proporción de trabajadores en la zona urbana es menor. Esto sugiere que existen elementos de composición productiva interna de las zonas que podrían explicar este resultado, aspecto que se retomará más adelante.

\section{Las brechas salariales por rama industrial}

El tercer elemento que ayuda a explicar una mayor desigualdad en el resto de los países centroamericanos, en comparación con Costa Rica, es el conjunto de variables binarias que dan cuenta de las diferencias de remuneración entre las distintas industrias. La desigualdad causada por las brechas de remuneración entre industrias es menor en Costa Rica que en el resto de la subregión. Las diferencias en las brechas salariales interindustriales se deben principalmente a las diferencias de remuneraciones entre la agricultura y el resto de las industrias, lo que sugiere que el análisis y la medición del efecto de las brechas salariales entre industrias sobre las diferencias de desigualdad deberían tomar en cuenta la interacción de las brechas salariales entre las industrias y las zonas de residencia. Desgraciadamente, la descomposición de Fields no permite medir adecuadamente las interacciones entre variables. Por lo tanto, para examinar tales interacciones, incluyendo la del tamaño de las empresas, se examinarán los coeficientes de una ecuación de remuneración que las expresa. ${ }^{20}$

En el cuadro 10 se presentan dichos coeficientes, así como el porcentaje de trabajadores en cada categoría. Se han definido ocho categorías en que interactúan la zona, el tamaño de la empresa y la rama industrial: i) trabajadores de pequeñas fincas (incluidos empleados, patronos o dueños y trabajadores por cuenta propia); ii) empleados de fincas grandes; iii) patronos o propietarios de fincas grandes; iv) trabajadores (incluidos empleados, patronos y trabajadores por cuenta propia) de pequeñas empresas rurales no agrícolas; v) empleados de grandes empresas rurales no agrícolas; vi) patronos o propietarios de grandes empresas rurales no agrícolas; vii) trabajadores (incluidos empleados, patronos y trabajadores por cuenta propia) de pequeñas empresas urbanas, y viii) trabajadores de grandes empresas urbanas.

Los coeficientes de las variables binarias presentados (cuadro 10) provienen de una ecuación de remuneración, la que también incluye las variables educación, sexo, logaritmo de las horas trabajadas, sector y experiencia. La categoría omitida es la de los trabajadores de las grandes empresas urbanas. De este modo, los coeficientes pueden ser interpretados como la diferencia porcentual entre las remuneraciones de los trabajadores de cada categoría y las remuneraciones de los trabajadores de las grandes empresas urbanas. Como se observa en el cuadro 10, en todos los países de la subregión los empleados de grandes empresas no

\footnotetext{
${ }^{20}$ La contribución conjunta a las diferencias en la desigualdad entre Costa Rica y el resto de los países centroamericanos de las brechas salariales de zona, tamaño e industria es más grande que el efecto de las brechas salariales por educación en todos los países, menos uno.
} 
CUADRO 10

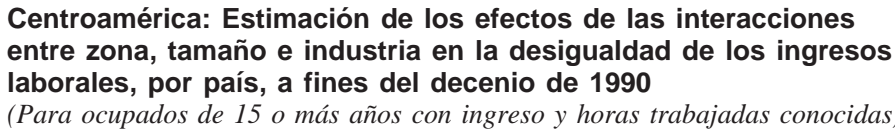

\begin{tabular}{|c|c|c|c|c|c|}
\hline Variables & $\begin{array}{c}\text { Costa Rica } \\
1999\end{array}$ & $\begin{array}{c}\text { El Salvador } \\
1999\end{array}$ & $\begin{array}{c}\text { Guatemala } \\
1998\end{array}$ & $\begin{array}{c}\text { Honduras } \\
1999\end{array}$ & $\begin{array}{c}\text { Nicaragua } \\
1998\end{array}$ \\
\hline \multicolumn{6}{|l|}{ Coeficientes de los distintos tipos de trabajadores ${ }^{a}$} \\
\hline Trabajadores de pequeñas fincas & $-0,51$ & $-0,59$ & $-0,74$ & $-0,95$ & $-0,88$ \\
\hline Empleados de fincas grandes & $-0,15$ & $-0,50$ & $-0,49$ & $-0,38$ & $-0,44$ \\
\hline Propietarios de fincas grandes & 0,22 & 0,91 & 2,28 & $-0,87$ & 0,39 \\
\hline Trabajadores de pequeñas empresas rurales no agrícolas & $-0,31$ & $-0,46$ & $-0,56$ & $-0,68$ & $-0,26$ \\
\hline Empleados de grandes empresas rurales no agrícolas & $-0,05$ & $-0,16$ & $-0,30$ & $-0,17$ & $-0,11$ \\
\hline Propietarios de grandes empresas rurales no agrícolas & 0,34 & 1,16 & 1,19 & 0,88 & \\
\hline Trabajadores de pequeñas empresas urbanas & $-0,24$ & $-0,27$ & $-0,31$ & $-0,34$ & $-0,16$ \\
\hline \multicolumn{6}{|l|}{ Porcentaje de trabajadores en cada categoría } \\
\hline Todos los trabajadores & 100,0 & 100,0 & 100,0 & 100,0 & 100,0 \\
\hline Trabajadores en actividades agrícolas & 17,1 & 9,8 & 28,6 & 25,6 & 21,6 \\
\hline Trabajadores de pequeñas fincas & 10,1 & 3,9 & 16,7 & 21,9 & 14,3 \\
\hline Empleados de fincas grandes & 6,8 & 5,9 & 11,7 & 3,6 & 7,0 \\
\hline Propietarios de fincas grandes & 0,1 & 0,0 & 0,2 & 0,2 & 0,4 \\
\hline Trabajadores rurales en actividades no agrícolas & 33,9 & 21,3 & 23,4 & 22,3 & 16,9 \\
\hline Trabajadores de pequeñas empresas rurales no agrícolas & 17,8 & 13,1 & 18,2 & 15,6 & 11,3 \\
\hline Empleados de grandes empresas rurales no agrícolas & 15,8 & 7,8 & 5,1 & 6,7 & 5,6 \\
\hline Propietarios de grandes empresas rurales no agrícolas & 0,3 & 0,4 & 0,1 & 0,0 & 0,0 \\
\hline Trabajadores de empresas urbanas & 49,0 & 68,9 & 48,0 & 52,1 & 61,5 \\
\hline Trabajadores de pequeñas empresas urbanas & 21,1 & 32,5 & 30,4 & 26,8 & 34,8 \\
\hline Trabajadores de grandes empresas urbanas & 27,9 & 36,5 & 17,6 & 25,3 & 26,7 \\
\hline
\end{tabular}

Fuente: Cálculos de los autores con base en las encuestas de hogares de los países y años respectivos.

a Diferencia porcentual entre las remuneraciones de cada tipo de trabajador y la de los trabajadores de las grandes empresas urbanas.

agrícolas de zonas rurales ganan menos que los trabajadores de las grandes empresas urbanas, pero más que cualquier tipo de trabajador de las zonas rurales. Asimismo, los empleados de las grandes fincas ganan menos que los trabajadores de las grandes empresas rurales no agrícolas, pero más que los trabajadores de las fincas pequeñas. Excepto en Nicaragua, los empleados de fincas grandes ganan más que los trabajadores de pequeñas empresas rurales no agrícolas. Y los trabajadores de las fincas pequeñas (empleados y dueños) ganan menos que cualquier otro grupo.

En todos los países centroamericanos, excepto Honduras, los propietarios de las grandes empresas rurales, agrícolas y no agrícolas, ganan más que los trabajadores de las grandes empresas urbanas. No obstante, la proporción de propietarios en el total de trabajadores es reducida, de modo que es poco probable que estos influyan mucho en las diferencias globales entre las remuneraciones urbanas y rurales. En todos los países la proporción de trabajadores rurales en activi- dades no agrícolas es alta, y en Costa Rica y El Salvador supera la proporción de trabajadores rurales en actividades agrícolas.

Estos resultados nos permiten comprender por qué la brecha salarial urbano-rural es menor en Costa Rica que en los otros países de la subregión. ${ }^{21}$ Primero, la brecha salarial entre trabajadores de grandes empresas rurales no agrícolas y grandes empresas urbanas es más pequeña en Costa Rica que en cualquier otro país. Segundo, la proporción de trabajadores en las grandes empresas rurales no agrícolas, que constituyen el sector rural mejor pagado, es más alta en Costa Rica que en

\footnotetext{
${ }^{21}$ En Guatemala fue posible identificar si los trabajadores pertenecían a un grupo indígena. Aunque la discriminación laboral contra los indígenas contribuyó a aumentar la desigualdad tanto en 1989 como en 1998, esta discriminación no explica las brechas salariales entre zonas urbanas y rurales. Agregar una variable que indique si el trabajador es indígena o no, no reduce la contribución a la desigualdad de la zona, el tamaño de la empresa o la rama industrial.
} 
cualquier otro país centroamericano. Tercero, la brecha salarial entre los trabajadores de las grandes empresas urbanas y los trabajadores de las empresas agrícolas, pequeñas o grandes, es más reducida en Costa Rica que en cualquier otro país de la subregión. Y cuarto, la proporción de trabajadores en pequeñas fincas es más bajo en Costa Rica que en el resto de Centroamérica.

Se ha señalado que El Salvador pertenece al segundo bloque, con una desigualdad mayor que la de Costa Rica, pero menor que la de Guatemala, Honduras y Nicaragua. ¿Por qué Guatemala, Honduras y Nicaragua muestran las mayores desigualdades en la distribución de los ingresos del trabajo? En gran parte porque el residuo, o la desigualdad debida a factores no observados, es mucho más alta en estos países que en Costa Rica y El Salvador (cuadro 9, primer bloque). Así por ejemplo, la desigualdad debida al residuo (factores no medidos) puede explicar por sí sola por qué la desigualdad es más acentuada en Nicaragua que en Costa Rica y El Salvador. El impacto del residuo en las ecuaciones de remuneración miden la parte de la desigualdad causada por variables que no han sido incluidas en la ecuación. Uno de los factores no incluidos son las diferencias no medidas entre las encuestas de hogares de los países. Las diferencias en el diseño de las muestras, en la forma de definir y medir las variables utilizadas — particularmente la del ingreso laboral—, en la calidad de la información recolectada, en la extensión de la no respuesta, etc., son aspectos que se pueden reflejar en el residuo. También es posible que las diferencias en los sistemas de salarios mínimos puedan explicar diferencias debidas al residuo, si los salarios mínimos legales tienen mayor cobertura y se aplican mejor en Costa Rica y El Salvador que en los demás países centroamericanos. ${ }^{22}$ También es posible que la desigualdad asociada con los grupos indígenas, que no se puede medir en la mayoría de los países, sea más grande en Guatemala, Honduras y Nicaragua.

Por otra parte, en Honduras tres fenómenos contribuyen a la gran desigualdad. En primer lugar, el premio salarial que perciben los trabajadores urbanos, o la brecha salarial urbano-rural, es el más alto entre todos los países de la subregión (apéndice A); su impacto es tal, que si la desigualdad se mide sólo para los trabajadores urbanos (cuadro 2), Honduras apare-

\footnotetext{
22 Aunque sólo Costa Rica ajusta los salarios mínimos legales periódicamente, cada semestre, estos dos países tienen los salarios mínimos más altos de la región.
}

ce con un grado de desigualdad similar al de El Salvador. En segundo lugar, el premio salarial recibido por los trabajadores de las empresas de mayor tamaño o formales es más alto en Honduras que en cualquier otro país centroamericano. Mucho de este resultado puede deberse a una brecha de remuneraciones entre trabajadores asalariados y trabajadores independientes (patronos o trabajadores por cuenta propia). Efectivamente, si se considera sólo los trabajadores asalariados (cuadro 2) la desigualdad en Honduras vuelve a parecerse a la de El Salvador. Por último, la brecha salarial entre hombres y mujeres es más grande en Honduras que en los otros países (apéndice A), y como está controlada por otras características mensurables del capital humano y del mercado de trabajo, se la puede interpretar como una medida burda de la discriminación contra las mujeres en el mercado de trabajo.

Guatemala, por su parte, explica su también alta desigualdad en los ingresos del trabajo por tres factores. Primero, la brecha salarial urbano-rural es más alta que en el resto de la subregión, excepto Honduras. Segundo, la brecha salarial entre hombres y mujeres es también una de las más altas, sólo superada por Honduras (apéndice). Finalmente, la desigualdad asociada con el número de horas trabajadas es similar a la de Costa Rica y más acentuada que la de cualquier otro país (cuadro 3). Esto sucede porque Guatemala y Costa Rica tienen el coeficiente más elevado de la variable logaritmo de horas trabajadas (apéndice); es decir, las remuneraciones de los trabajadores aumentan más rápidamente al trabajar horas adicionales que en el resto de los países centroamericanos, y la desigualdad causada por la distribución de las horas trabajadas también es muy alta (cuadro 9).

Finalmente, cabe destacar dos aspectos. En primer lugar, que las conclusiones de este estudio están limitadas a las que se pueden justificar con la información provista por las encuestas de hogares. Es posible que haya otras variables que sean causas importantes de las diferencias en la desigualdad entre países. En ese sentido, se ha señalado que la brecha salarial entre indígenas y no indígenas es una importante fuente de desigualdad en Guatemala, pero no se puede establecer la comparación con el resto de los países centroamericanos. En un estudio reciente del Banco Mundial (de Ferrari, Perry y otros, 2004) se presenta evidencia de que las diferencias entre países de la brecha salarial entre indígenas y no indígenas explican una parte significativa de las diferencias de desigualdad entre ellos. En segundo lugar, la información y el análisis se circunscriben al decenio 
de 1990, de modo que no es posible llegar a conclusiones sobre las razones históricas de los patrones observados. Por ejemplo, se ha encontrado que la menor desigualdad relativa de Costa Rica se sustenta en una distribución más equitativa de la educación y en una menor brecha salarial entre zonas urbanas y rurales. Esto se ha asociado con una mayor y más equi- tativa inversión en infraestructura y en la provisión de servicios sociales. El citado estudio del Banco Mundial concuerda con estas razones y las vincula con raíces históricas en la estructura socioeconómica y la transición política de fines del siglo XIX. Razones históricas opuestas explicarían los mayores niveles de desigualdad en Guatemala.

\section{VI}

\section{Conclusiones de política}

En la década de 1990, la dirección de los cambios en la desigualdad del ingreso laboral en los países centroamericanos no fue uniforme y determinarla depende del indicador de desigualdad que se aplique. Sólo Costa Rica y Guatemala presentan un deterioro claro en la distribución del ingreso del trabajo. Pero bajo esos cambios brutos se encuentran fenómenos comunes a todos los países en los mercados de trabajo. El fenómeno que ha tenido el efecto adverso más importante sobre la distribución del ingreso laboral es la reducción del porcentaje de trabajadores con jornada completa en cada país y el aumento concomitante de los trabajadores con jornada parcial y con sobrejornada. Este cambio aumentó la dispersión entre los trabajadores del número de horas trabajadas, lo que acentuó la desigualdad del ingreso laboral. La disminución de trabajadores con jornada completa refleja la reducción del empleo en el sector público en todos los países de la subregión. Por su parte, el aumento de trabajadores con jornada parcial refleja la ascendente proporción de mujeres en la fuerza de trabajo y la importancia de las empresas pequeñas privadas (el sector informal) como fuente de empleo, mientras que el incremento de los trabajadores con sobrejornadas se asocia con empresas de mayor tamaño y sus esfuerzos por mejorar la productividad y rentabilidad.

En este trabajo se investigaron también las diferencias entre los países centroamericanos y, en especial, por qué Costa Rica tiene un nivel de desigualdad menor que los demás. Una causa importante de este fenómeno es que en Costa Rica la educación está más equitativamente distribuida. Almeida dos Reas y Paes de Barros (1991), que presentaron un modelo de expansión educativa y desigualdad, concluyen que la expansión educativa reduce la desigualdad si ocurre entre niveles educativos en los cuales los trabajadores ganan menos que el in- greso medio. Pero que si se concentra en niveles educativos en los cuales los trabajadores ganan más que el salario medio, como la educación universitaria, esa expansión contribuye a incrementar la desigualdad. ${ }^{23}$ Esto sugiere que las políticas de universalización de la educación primaria aplicadas por Costa Rica en los decenios de 1960 y 1970 fueron una importante causa de los diferentes niveles de desigualdad encontrados. Esas políticas bajaron la proporción de trabajadores sin educación o con enseñanza primaria incompleta e incrementaron la proporción de trabajadores con educación primaria completa (a todos estos niveles los trabajadores ganan salarios inferiores al ingreso medio). Asimismo, en Costa Rica y El Salvador la rentabilidad (o precio) de la educación es menor que en Guatemala, Honduras y Nicaragua, países con niveles educativos muy bajos. Esto sugiere que la mayor oferta relativa de trabajadores con mayor nivel educativo en Costa Rica y El Salvador contribuye a reducir el precio relativo de la educación y, en esa medida, a mejorar la distribución de los ingresos.

Se ha encontrado también que las diferencias salariales entre las zonas urbanas y rurales son más bajas en Costa Rica porque en este país los trabajadores de las grandes empresas rurales no agrícolas (presumiblemente de alta productividad) ganan más y son una mayor proporción de los trabajadores rurales que en el resto de

\footnotetext{
${ }^{23}$ Por otro lado, la expansión de la educación que ocurre entre niveles de educación superiores al promedio (o sea, la que incrementa la proporción de trabajadores que ganan más que el salario medio y baja la proporción de trabajadores que ganan menos que el salario medio) puede aumentar o disminuir la desigualdad. Este tipo de expansión educativa incrementará la desigualdad si hay pocos trabajadores de nivel educativo alto y bajará la desigualdad si hay muchos trabajadores de nivel educativo alto. Esto es un ejemplo de la famosa Curva de Kuznets.
} 
Centroamérica. Tales resultados son consistentes con los resultados y las recomendaciones de política contenidos en López y Valdés (2000). En dicho trabajo, que resume estudios sobre la pobreza rural en varios países de América Latina, incluidos El Salvador, Guatemala y Honduras, se presenta evidencia de que las grandes empresas rurales pagan más que otros empleos rurales, y que además los trabajadores rurales en América Latina son menos dependientes de la economía agrícola que en casi cualquier otro lugar del mundo en desarrollo; asimismo, se sugiere que para reducir la pobreza rural, la mayor parte de los recursos públicos debería dedicarse a mejorar las remuneraciones y las oportunidades laborales en empleos no agrícolas en las zonas rurales, y se dan pruebas de que existe una correlación entre la proporción de empleos rurales no agrícolas de alta productividad, por un lado, y mayores niveles medios de educación y mejor infraestructura rural (como carreteras, electricidad, teléfonos), por otro. Lo dicho sugiere que las políticas públicas costarricenses de suministrar hasta a las comunidades rurales más aisladas servicios de electricidad, teléfonos, educación, salud e infraestructura de transporte (todos altamente relacionados con actividades rurales no agrícolas de alta productividad), es también causa importante de las diferencias en la desigualdad entre Costa Rica y el resto de la subregión y da luces sobre políticas públicas que buscan reducir la pobreza y la desigualdad.

APENDICE

Centroamérica: Estadísticos básicos de las ecuaciones de remuneración, por país, a fines del decenio de 1990

(Para ocupados de 15 o más años con ingreso y horas trabajadas conocidas)

\begin{tabular}{|c|c|c|c|c|c|c|c|c|c|c|}
\hline \multirow[t]{2}{*}{ Variables } & \multicolumn{2}{|c|}{ Costa Rica } & \multicolumn{2}{|c|}{ El Salvador } & \multicolumn{2}{|c|}{ Guatemala } & \multicolumn{2}{|c|}{ Honduras } & \multicolumn{2}{|c|}{ Nicaragua } \\
\hline & 1990 & 1999 & 1995 & 1999 & 1989 & 1998 & 1990 & 1999 & 1993 & 1998 \\
\hline \multicolumn{11}{|l|}{$\begin{array}{l}\text { Coeficientes de las ecuaciones } \\
\text { de remuneración }\end{array}$} \\
\hline Educación (años) & 0,087 & 0,089 & 0,064 & 0,081 & 0,093 & 0,112 & 0,116 & 0,106 & 0,087 & 0,094 \\
\hline Sexo $($ hombre $=1)$ & 0,278 & 0,270 & 0,307 & 0,241 & 0,313 & 0,464 & 0,479 & 0,469 & 0,136 & 0,323 \\
\hline Zona (urbano $=1)$ & 0,087 & 0,060 & 0,255 & 0,169 & 0,156 & 0,243 & 0,257 & 0,313 & 0,312 & 0,156 \\
\hline Horas trabajadas (logaritmo) & 0,573 & 0,571 & 0,421 & 0,423 & 0,488 & 0,562 & 0,353 & 0,415 & 0,244 & 0,172 \\
\hline Sector institucional (público $=1$ ) & 0,233 & 0,211 & 0,288 & 0,439 & 0,166 & 0,085 & 0,237 & 0,175 & $-0,102$ & $-0,164$ \\
\hline Tamaño establecimiento $(6$ o más $=1$ ) & 0,273 & 0,270 & 0,252 & 0,283 & 0,470 & 0,325 & 0,385 & 0,426 & 0,440 & 0,272 \\
\hline Experiencia (edad-educación-6) & 0,033 & 0,027 & 0,030 & 0,037 & 0,033 & 0,054 & 0,046 & 0,039 & 0,039 & 0,041 \\
\hline Experiencia al cuadrado & $-0,0004$ & $-0,0003$ & $-0,0004$ & $-0,0005$ & $-0,0004$ & $-0,0007$ & $-0,0005$ & $-0,0004$ & $-0,0005$ & $-0,0005$ \\
\hline $\begin{array}{l}\text { Industria (conjunto varias binarias } \\
\text { por rama) }\end{array}$ & sig & sig & sig & sig & sig & sig & sig & sig & sig & sig \\
\hline $\mathrm{R}^{2}$ & 0,486 & 0,503 & 0,460 & 0,494 & 0,441 & 0,443 & 0,450 & 0,450 & 0,387 & 0,324 \\
\hline Número de observaciones & 9.704 & 13.152 & 10.365 & 19.824 & 12.747 & 11.615 & 12.293 & 10.778 & 4.806 & 5.978 \\
\hline
\end{tabular}

Desviación estándar de las

variables independientes

Educación (años)

Sexo (hombre $=1)$

Zona (urbano $=1$ )

Horas trabajadas (logaritmo)

Sector institucional (público $=1$ )

Tamaño establecimiento $(6$ o más $=1)$

Experiencia (edad-educación-6)

4,06

\section{0,45}

0,50

0,46

0,39

0,50

14,36

4,06
0,47
0,50
0,56
0,34
0,50
14,12

5,14
0,49
0,47
0,41
0,32
0,50
15,68

\section{5,03}

0,50

0,46

0,47

0,31

0,50

16,00

$\begin{array}{rr}4,33 & 4,61 \\ 0,44 & 0,48 \\ 0,50 & 0,50 \\ 0,35 & 0,58 \\ 0,28 & 0,23 \\ 0,49 & 0,48 \\ 16,25 & 17,20\end{array}$

\section{4,21}

0,46

0,50

0,47

0,30

0,48

16,23

4,47
0,48
0,50
0,56
0,27
0,48
16,26

4,64

0,49

0,48

0,58

0,39

0,49

15,15

4,48

0,70

0,48

3,77

0,10

$\begin{array}{rrrr}0,50 & 0,39 & 0,35 & 0,35 \\ 23,27 & 26,57 & 26,80 & 25,80\end{array}$

$\begin{array}{rrrr}0,50 & 0,39 & 0,35 & 0,35 \\ 23,27 & 26,57 & 26,80 & 25,80\end{array}$

$\begin{array}{ll}7,33 & 7,81 \\ 0,71 & 0,68 \\ 0,46 & 0,49 \\ 3,76 & 3,74 \\ 0,18 & 0,13 \\ 0,57 & 0,51\end{array}$

$20,49 \quad 22,86$

3,77

0,11

24,10

7,01
0,56
0,69
3,72
0,11
0,50
23,27

5,45

0,63

0,52

3,74

0,08

0,36

24,57
5,47

0,62

0,64

3,74

0,19

0,40

24,29
4,66

0,48

0,49

0,55

0,32

0,49

15,52

Fuente: Cálculos de los autores con base en las encuestas de hogares de los países y años respectivos. 


\section{Bibliografía}

Autor, D. y L. Katz (1999): Changes in the wage structure and earnings inequality, en O. Ashenfelter y D. Card (comps.), Handbook of Labor Economics, vol. 3A, Nueva York, NorthHolland.

Almeida dos Reas, J.G. y R. Paes de Barros (1991): Wage inequality and the distribution of education: a study of the evolution of regional differences in inequality in metropolitan Brazil, Journal of Development Economics, vol. 36, № 1, Amsterdam, Elsevier Science, julio.

CEPAL (Comisión Económica para América Latina y el Caribe) (2001): Panorama social de América Latina, 2000-2001, LC/ G.2138-P, Santiago de Chile, septiembre. Publicación de las Naciones Unidas, $\mathrm{N}^{\circ}$ de venta: S.01.II.G.141.

(2002): Panorama social de América Latina, 2001-2002, LC/G.2183-P, Santiago de Chile, octubre. Publicación de las Naciones Unidas, $\mathrm{N}^{\circ}$ de venta: S.02.II.G.65.

(2003): Anuario estadístico de América Latina y el Caribe, 2002, LC/G.2190-P, Santiago de Chile. Publicación de las Naciones Unidas, $\mathrm{N}^{\mathrm{o}}$ de venta: E/S.03.II.G.1.

Contreras, D. (2003): Explaining wage inequality in Chile, Journal of Income Distribution, vol. 11, № 1-2, Amsterdam, Elsevier Science.

De Ferrari, D., G. Perry y otros (2004): Inequality in Latin America and the Caribbean: Breaking with History, Washington, D.C., Banco Mundial.

Fields, G.S. (2003): Accounting for income inequality and its change: a new method, with application to the distribution of earnings in the United States, en S. Polachek (comp.), Research in Labor Economics, vol. 22, Amsterdam, Elsevier Science.

Fields, G.S. e I. Gyeongjoon (2000): Falling labor income inequality in Korea's economic growth: patterns and underlying causes, Review of Income and Wealth, vol. 46, $\mathrm{N}^{\circ} 1$, Nueva York, Universidad de Nueva York, junio.

Ganuza, E., R. Paes de Barros y otros (comps.) (2001): Liberalización, desigualdad y pobreza: América Latina y el Caribe en los 90, Buenos Aires, EUDEBA.

Gindling, T.H. y J.D. Trejos (2004): Accounting for changing earnings inequality in Costa Rica, 1980-1999, Journal of Development Studies, Amsterdam, Elsevier Science, por aparecer.

Juhn, C., K. Murphy y B. Pierce (1993): Wage inequality and the rise in returns to skill, Journal of Political Economy, vol. 101, $\mathrm{N}^{\mathrm{o}} 3$, Chicago, University of Chicago Press.

Katz, L. y K. Murphy (1992): Changes in relative wages, 19631987: supply and demand factors, Quarterly Journal of
Economics, vol. 107, Cambridge, Massachusetts, Harvard University.

Koujianou Goldberg, P. y N. Pavcnik (2001): Trade, Wages, and the Political Economy of Trade Protection: Evidence from the Colombian Trade Reforms, Hanover, NH, Dartmouth College, noviembre, inédito.

López, R. y A. Valdés (2000): Fighting rural poverty in Latin America: new evidence of the effects of education, demographics, and access to land, Economic Development and Cultural Change, vol. 49, No 1 , Chicago, University of Chicago Press, octubre.

Lora, E. (2001): Structural Reforms in Latin America: What Has Been Reformed and How to Measure It, Research Department Working Paper, $N^{\circ} 466$, Washington, D.C., Banco Interamericano de Desarrollo (BID), diciembre.

Morley, S., R. Machado y S. Pettinato (1999): Indexes of Structural Reform in Latin America, serie Reformas económicas, $\mathrm{N}^{\mathrm{o}}$ 12, LC/L.1166, Santiago de Chile, Comisión Económica para América Latina y el Caribe (CEPAL).

PNUD (Programa de las Naciones Unidas para el Desarrollo) (2002): Informe sobre desarrollo humano, 2002: profundizar la democracia en un mundo fragmentado, Madrid, Mundi-Prensa Libros.

Robertson, R. (1999): Inter-Industry Wage Differentials across Time, Borders, and Trade Regimes: Evidence from the U.S. and Mexico, St. Paul, Macalester College, inédito.

Shorrocks, A. (1982): Inequality decomposition by factor components, Econometrica, vol. 50, No 1 , Evanston, Illinois, The Econometric Society, enero.

Székely, M. y M. Hilgert (1999a): The 1990s in Latin America: Another Decade of Persistent Inequality, Research Department Working Paper, $N^{\circ} 410$, Washington, D.C., Banco Interamericano de Desarrollo (BID), diciembre.

(1999b): What's Behind the Inequality We Measure: An Investigation Using Latin American Data for the 1990s, Bank Research Department, Washington, D.C., Banco Interamericano de Desarrollo (BID), diciembre.

Trejos, J.D. (2002): El trabajo decente y el sector informal en los países del istmo centroamericano, documento $\mathrm{N}^{\circ} 158$, San José, Organización Internacional del Trabajo, Oficina para Centroamérica, Panamá y República Dominicana.

Yun, M. (2002): Earnings Inequality in the USA, 1961-1999: Comparing Inequality Using Earnings Equations, Tulane University, inédito. 